\title{
Abstract Functional Stochastic Evolution Equations Driven by Fractional Brownian Motion
}

\author{
Mark A. McKibben ${ }^{1}$ and Micah Webster ${ }^{2}$ \\ ${ }^{1}$ Department of Mathematics, West Chester University of Pennsylvania, 25 University Avenue, West Chester, PA 19383, USA \\ ${ }^{2}$ Department of Mathematics and Computer Science, Goucher College, 1021 Dulaney Valley Road, Baltimore, MD 21204, USA
}

Correspondence should be addressed to Mark A. McKibben; mmcKibben@wcupa.edu

Received 21 October 2013; Revised 15 December 2013; Accepted 20 December 2013; Published 25 February 2014

Academic Editor: T. Diagana

Copyright ( 2014 M. A. McKibben and M. Webster. This is an open access article distributed under the Creative Commons Attribution License, which permits unrestricted use, distribution, and reproduction in any medium, provided the original work is properly cited.

\begin{abstract}
We investigate a class of abstract functional stochastic evolution equations driven by a fractional Brownian motion in a real separable Hilbert space. Global existence results concerning mild solutions are formulated under various growth and compactness conditions. Continuous dependence estimates and convergence results are also established. Analysis of three stochastic partial differential equations, including a second-order stochastic evolution equation arising in the modeling of wave phenomena and a nonlinear diffusion equation, is provided to illustrate the applicability of the general theory.
\end{abstract}

\section{Introduction}

The purpose of this paper is to study the global existence and convergence properties of mild solutions to a class of abstract functional stochastic evolution equations of the general form

$$
\begin{gathered}
d x(t)=(A x(t)+\mathscr{F}(x)(t)) d t+g(t) d \beta^{H}(t), \\
0 \leq t \leq T, \\
x(0)=x_{0},
\end{gathered}
$$

in a real separable Hilbert space $U$. Here, $A: D(A) \subset U \rightarrow U$ is a linear (possibly unbounded) operator which generates a strongly continuous semigroup $\{S(t): t \geq 0\}$ on $U$; $\mathscr{F}: \mathbb{C}\left([0, T] ; \mathscr{L}^{2}(\Omega ; U)\right) \rightarrow \mathscr{L}^{2}\left((0, T) ; \mathscr{L}^{2}(\Omega ; U)\right)$ is a given mapping; $g:[0, T] \rightarrow(V ; U)$ is a bounded, strongly measurable mapping (where $V$ is a real separable Hilbert space and $\mathfrak{L}(V ; U)$ denotes the space of Hilbert-Schmidt operators from $V$ into $U$ with norm $\|\cdot\|_{\mathcal{Q}(V ; U)}$ equipped with the strong topology); $\left\{\beta^{H}(t): t \geq 0\right\}$ is a $U$-valued fBm with Hurst parameter $H \in(1 / 2,1)$; and $x_{0} \in \mathscr{L}_{0}^{2}(\Omega ; U)$.

Stochastic partial functional differential equations naturally arise in the mathematical modeling of phenomena in the natural sciences (see [1-6]). It has been shown that some applications, such as communication networks and certain financial models, exhibit a self-similarity property in the sense that the processes $\{x(\alpha t): 0 \leq t \leq T\}$ and $\left\{\alpha^{H} x(t)\right.$ : $0 \leq t \leq T\}$ have the same law (see $[4,7]$ ). Concrete data from a variety of applications have exhibited behavior that differs from standard Brownian motion $(H=1 / 2)$, and it seems that these differences enter in a nonnegligible way in the modeling of this phenomena. In fact, since $\beta^{H}(t)$ is not a semimartingale unless $H=1 / 2$, the standard stochastic calculus involving the Itó integral cannot be used in the analysis of related stochastic evolution equations. There have been several papers devoted to the formulation of stochastic calculus for $\mathrm{fBm}\left[\mathrm{8}^{-11}\right]$ and differential/evolution equations driven by $\mathrm{fBm}$ [12-14] published in the past decade. We provide an outline of only the necessary concomitant technical details concerning the construction of the stochastic integral driven by an $\mathrm{fBm}$ and some of its properties in Section 2 .

The present work may be regarded as a direct attempt to extend results developed in $[1,12,15-18]$ to a broader class of functional stochastic equations. The equations considered in the aforementioned papers can be viewed as special cases of (1) by appropriately defining the functional $\mathscr{F}$, the correct space $U$, and the appropriate value of $H$. In particular, the existence and convergence results we present constitute 
generalizations of the theory governing standard models arising in the mathematical modeling of nonlinear diffusion processes [1, 15, 18-22] and communication networks [4].

The outline of the paper is as follows. We collect some preliminary information about certain function spaces, linear semigroups, probability measures, the definition of $\mathrm{fBm}$, and the stochastic integral driven by a fBmin Section 2. The main existence results in the Lipschitz and compactness cases are discussed in Section 3, while convergence results are developed in Section 4. An extension of an existence result of the case of second-order stochastic evolution equations is discussed in Section 5. The paper concludes with a discussion of three different stochastic partial differential equations in Section 6 as an illustration of the abstract theory.

\section{Preliminaries}

For further background of this section, we refer the reader to $[6,8,9,12,23-28]$ and the references therein. Throughout this paper, $U$ is a real separable Hilbert space with norm $\|\cdot\|_{U}$ and inner product $\langle\cdot, \cdot\rangle_{U}$ equipped with a complete orthonormal basis $\left\{e_{j} \mid j=1,2, \ldots\right\}$, and $(\Omega, \mathscr{G}, P)$ is a complete probability space. We suppress the dependence of all random variables on $\omega \in \Omega$ throughout the manuscript and write $x(t)$ instead of $x(t ; \omega)$.

We make use of several different function spaces throughout this paper. The space of all bounded linear operators on $U$ is denoted by $\mathfrak{B} \mathfrak{Q}(U)$, while $\mathscr{L}^{2}(\Omega ; U)$ stands for the space of all $U$-valued random variables $y$ for which $E\|y\|_{U}^{2}<\infty$, where the expectation, $E$, is defined by $E(g)=\int_{\Omega} g(\omega) d P$. An important subspace is given by

$$
\begin{aligned}
& \mathscr{L}_{0}^{2}(\Omega ; U) \\
& \quad\left\{f \in \mathscr{L}^{2}(\Omega ; U): f \text { is }\left(\mathscr{G}_{0}, \mathscr{B}(U)\right) \text {-measurable }\right\},
\end{aligned}
$$

where $\left\{\mathscr{G}_{t}: 0 \leq t \leq T\right\}$ is the family of $\sigma$-algebras $\mathscr{G}_{t}$ generated by $\left\{\beta^{H}(s): 0 \leq s \leq t\right\}$ and $\mathscr{B}(U)$ is the Borel class on $U$. The space of $\mathscr{L}^{2}$-continuous $U$-valued random variables $y:[0, T] \rightarrow U$ such that

$$
\|y\|_{\mathbb{C}}^{2} \equiv\|y\|_{\mathbb{C}\left([0, T] ; \mathscr{L}^{2}(\Omega ; U)\right)}^{2}=\sup _{0 \leq t \leq T} E\|y(t)\|_{U}^{2}<\infty
$$

is denoted by $\mathbb{C}\left([0, T] ; \mathscr{L}^{2}(\Omega ; U)\right)$.

The following alternative of the Leray-Schauder principle [29] plays a role in Section 3.

Theorem 1. Let $X$ be a Banach space, and let $\Phi: X \rightarrow X$ be a completely continuous map. Then, either $\Phi$ has a fixed point, or the set $\xi(\Phi)=\{x \in X: \lambda x=\Phi x$, for some $\lambda \geq 1\}$ is unbounded.

The probability measure $\bar{P}$ induced by a $U$-valued random variable $X: \Omega \rightarrow U$, denoted by $\overline{P_{X}}$, is defined by $\bar{P} \circ X^{-1}: \mathscr{B}(U) \rightarrow[0,1]$. A sequence $\left\{\overline{P_{n}}\right\} \subset \wp(U)$ is said to be weakly convergent to $\bar{P}$ if $\int_{\Omega} f d \overline{P_{n}} \rightarrow \int_{\Omega} f d \bar{P}$, for every bounded, continuous function $f: U \rightarrow \mathbb{R}$; in such case, we write $\overline{P_{n}} \stackrel{w}{\rightarrow} \bar{P}$. A family $\left\{\overline{P_{n}}\right\}$ is tight if for each $\varepsilon>0$, there exists a compact set $K_{\varepsilon}$ such that $\overline{P_{n}}\left(K_{\varepsilon}\right) \geq$ $1-\varepsilon$, for all $n$. Kunita [27] established the equivalence of tightness and relative compactness of a family of probability measures. Consequently, the Arzelá-Ascoli theorem can be used to establish tightness.

Definition 2. Let $\bar{P} \in \wp(U)$ and $0 \leq t_{1}<t_{2}<$ $\cdots<t_{k} \leq T$. Define $\pi_{t_{1}, \ldots, t_{k}}: \mathbb{C}\left([0, T] ; \mathscr{L}^{2}(\Omega ; U)\right) \rightarrow$ $U^{k}$ by $\pi_{t_{1}, \ldots, t_{k}}(X)=\left(X\left(t_{1}\right), \ldots, X\left(t_{k}\right)\right)$. The probability measures induced by $\pi_{t_{1}, \ldots, t_{k}}$ are the finite dimensional joint distributions of $\bar{P}$.

Proposition 3. If a sequence $\left\{X_{n}: \Omega \rightarrow U\right\}$ of $U$-valued random variables converges weakly to a $U$-valued random variable $X: \Omega \rightarrow U$ in the mean-square sense, then the sequence of finite dimensional joint distributions corresponding to $\left\{\overline{P_{X_{n}}}\right\}$ converges weakly to the finite dimensional joint distribution of $\overline{P_{X}}$.

The next theorem, in conjunction with Proposition 3, is the main tool used to prove one of the convergence results in this paper.

Theorem 4. Let $\left\{\overline{P_{n}}\right\} \subset \wp(U)$. If the sequence of finite dimensional joint distributions corresponding to $\left\{\overline{P_{n}}\right\}$ converges weakly to the finite dimensional joint distribution of $\bar{P}$ and $\left\{\overline{P_{n}}\right\}$ is relatively compact, then $\overline{P_{n}} \stackrel{w}{\rightarrow} \bar{P}$.

We next make precise the definition of a $U$-valued $\mathrm{fBm}$ and related stochastic integral used in this paper. The approach we use coincides with the one formulated and analyzed in $[12,30]$. Let $\left\{\beta_{j}^{H}(t) \mid t \geq 0\right\}_{j=1}^{\infty}$ be a sequence of independent, one-dimensional $\mathrm{fBms}$ with Hurst parameter $H \in(1 / 2,1)$ such that, for all $j=1,2, \ldots$,

(i) $\beta_{j}^{H}(0)=0$,

(ii) $E\left[\beta_{j}^{H}(t)-\beta_{j}^{H}(s)\right]^{2}=|t-s|^{2 H} v_{j}$,

(iii) $E\left[\beta_{j}^{H}(1)\right]^{2}=v_{j}>0$,

(iv) $\sum_{j=1}^{\infty} v_{j}<\infty$.

In such case, $\sum_{j=1}^{\infty} E\left\|\beta_{j}^{H}(t) e_{j}\right\|_{U}^{2}=t^{2 H} \sum_{j=1}^{\infty} v_{j}<\infty$, so that the following definition is meaningful.

Definition 5. For every $t \geq 0, \beta^{H}(t)=\sum_{j=1}^{\infty} \beta_{j}^{H}(t) e_{j}$ is a $U$ valued $\mathrm{fBm}$, where the convergence is understood to be in the mean-square sense.

It has been shown in $[12,30]$ that the covariance operator of $\left\{\beta^{H}(t): t \geq 0\right\}$ is a positive nuclear operator $Q$ such that

$$
\operatorname{tr} Q(t, s)=\frac{1}{2} \sum_{j=1}^{\infty} v_{j}\left[t^{2 H}+s^{2 H}-|t-s|^{2 H}\right] .
$$

Next, we outline the discussion leading to the definition of the stochastic integral associated with $\left\{\beta^{H}(t): t \geq 0\right\}$ 
for bounded, strongly measurable functions $g:[0, T] \rightarrow$ $\mathfrak{Q}(V ; U)$. To begin, assume that such a function $g$ is simple, meaning that there exists $\left\{g_{i}: i=1, \ldots, n\right\} \subseteq \mathfrak{Q}(V ; U)$ such that

$$
g(t)=g_{i}, \quad \forall t_{i-1} \leq t<t_{i}
$$

where $0=t_{0}<t_{1}<\cdots<t_{n-1}<t_{n}=T$ and $\max _{1 \leq i \leq n}\left\|g_{i}\right\|_{\mathfrak{Q}(V ; U)}=K$.

Definition 6. The $U$-valued stochastic integral $\int_{0}^{T} g(t) d \beta^{H}(t)$ is defined by

$$
\begin{aligned}
\int_{0}^{T} g(t) d \beta^{H}(t) & =\sum_{j=1}^{\infty}\left(\int_{0}^{T} g(t) d \beta_{j}^{H}(t)\right) e_{j} \\
& =\sum_{j=1}^{\infty}\left(\sum_{i=1}^{n} g_{i}\left[\beta_{j}^{H}\left(t_{i}\right)-\beta_{j}^{H}\left(t_{i-1}\right)\right]\right) e_{j} .
\end{aligned}
$$

As argued in Lemma 2.2 of [30], this integral is well defined since

$$
E\left\|\int_{0}^{T} g(t) d \beta^{H}(t)\right\|_{U}^{2} \leq K^{2} T^{2 H} \sum_{j=1}^{\infty} v_{j}<\infty
$$

Since the set of simple functions is dense in the space of bounded, strongly measurable $\mathfrak{Q}(V ; U)$-valued functions, a standard density argument can be used to extend Definition 6 to the case of a general bounded, strongly measurable integrand.

\section{Existence Results}

We consider mild solutions of (1) in the following sense.

Definition 7. A stochastic process $x \in \mathbb{C}\left([0, T] ; \mathscr{L}^{2}(\Omega ; U)\right)$ is a mild solution of (1) if

$$
\begin{array}{r}
x(t)=S(t) x_{0}+\int_{0}^{t} S(t-s) \mathscr{F}(x)(s) d s \\
+\int_{0}^{t} S(t-s) g(s) d \beta^{H}(s), \\
\forall 0 \leq t \leq T, \text { a.s. }[P] .
\end{array}
$$

For our first result, we impose the following conditions on (1):

(H1) $A: D(A) \subset U \rightarrow U$ is the infinitesimal generator of a strongly continuous semigroup $\{S(t): t \geq 0\}$ on $U$ such that $\|S(t)\|_{\mathfrak{B R}(U)} \leq M \exp (\alpha t)$, for all $0 \leq t \leq T$, for some $M \geq 1$ and $\alpha>0$;

$(\mathrm{H} 2) \mathscr{F}: \mathbb{C}\left([0, T] ; \mathscr{L}^{2}(\Omega ; U)\right) \rightarrow \mathscr{L}^{2}\left((0, T) ; \mathscr{L}^{2}(\Omega ; U)\right)$ is such that there exists a positive constant $M_{\mathscr{F}}$ for which

$$
\begin{aligned}
& \|\mathscr{F}(x)-\mathscr{F}(y)\|_{\mathscr{L}^{2}} \leq M_{\mathscr{F}}\|x-y\|_{\mathbb{C}} \\
& \quad \text { for all } x, y \in \mathbb{C}\left([0, T] ; \mathscr{L}^{2}(\Omega ; U)\right) ;
\end{aligned}
$$

(H3) $g:[0, T] \rightarrow \mathfrak{Q}(V ; U)$ is a bounded, strongly measurable mapping;

(H4) $\left\{\beta^{H}(t): t \geq 0\right\}$ is a $U$-valued $\mathrm{fBm}$;

(H5) $x_{0} \in \mathscr{L}_{0}^{2}(\Omega ; U)$.

(Henceforth, we write $M_{S}=\max _{0 \leq t \leq T}\|S(t)\|_{\mathfrak{B Q}(U)}$, which can be shown to be finite by using (H1) and the Uniform Boundedness Principle.)

The following technical properties involving the stochastic integral $\int_{0}^{t} S(t-s) g(s) d \beta^{H}(s)$, under assumptions (H1), $(\mathrm{H} 3)$, and ( $\mathrm{H} 4)$, are used in the proofs of the main results in this paper.

Lemma 8. Assume (H1), (H3), and (H4). Then, for all $0 \leq t \leq$ $T$,

(i) $E\left\|\int_{0}^{t} S(t-s) g(s) d \beta^{H}(s)\right\|_{U}^{2} \leq C_{t} \sum_{j=1}^{\infty} v_{j}$, (ii) $\lim _{h \rightarrow 0} E\left\|\int_{0}^{t+h}[S(t+h-s)-S(t-s)] g(s) d \beta^{H}(s)\right\|_{U}^{2}$
$\quad=0$.

Here, $C_{t}$ is a positive constant depending on $t,\{S(t): 0 \leq$ $t \leq T\}$, and $K\left(c f\right.$. (5)), and $\left\{v_{j}: j \in \mathbb{N}\right\}$ is defined as in the discussion leading to Definition 5.

Proof. Property (i) can be established as in Lemma 6 in [12]. To verify property (ii), let $0 \leq t \leq T$ and observe that

$$
\begin{aligned}
& E\left\|\int_{0}^{t}[S(t+h-s)-S(t-s)] g(s) d \beta^{H}(s)\right\|_{U}^{2} \\
& =E\left\|\sum_{j=1}^{\infty} \int_{0}^{t}[S(t+h-s)-S(t-s)] g(s) e_{j} d \beta_{j}^{H}(s)\right\|_{U}^{2} \\
& \leq 2 E\left\|\sum_{j=1}^{m} \int_{0}^{t}[S(t+h-s)-S(t-s)] g(s) e_{j} d \beta_{j}^{H}(s)\right\|_{U}^{2} \\
& +2 E\left\|\sum_{j=m+1}^{\infty} \int_{0}^{t}[S(t+h-s)-S(t-s)] g(s) e_{j} d \beta_{j}^{H}(s)\right\|_{U}^{2} .
\end{aligned}
$$

The strong continuity of $S(\cdot)$, together with $(\mathrm{H} 3)$, guarantees that the first term on the right side of (10) goes to zero as $h \rightarrow$ 0 . To argue the second term goes to zero, we first assume that $g$ is a simple function as defined in (5). Arguing as in [12] yields the estimate

$$
E\left\|\int_{0}^{t}[S(t+h-s)-S(t-s)] g(s) e_{j} d \beta_{j}^{H}(s)\right\|_{U}^{2} \leq C_{t} t^{2 h} v_{j}
$$


where $C_{t}$ is defined as in part (i) of this lemma. Using (11) in the second term on the right side of (10) yields

$$
\begin{gathered}
E\left\|\sum_{j=m+1}^{\infty} \int_{0}^{t}[S(t+h-s)-S(t-s)] g(s) e_{j} d \beta_{j}^{H}(s)\right\|_{U}^{2} \\
\leq \sum_{j=m+1}^{\infty} C_{t} t^{2 h} v_{j} .
\end{gathered}
$$

The convergence of $\sum_{j=1}^{\infty} v_{j}$ ensures that the right side of (12) goes to zero as $m \rightarrow \infty$. As such, property (ii) holds for a simple function $g$. It is not difficult to extend the argument to general bounded, strongly measurable functions $g$. This completes the proof.

Consider the solution map $\Phi: \mathbb{C}\left([0, T] ; \mathscr{L}^{2}(\Omega ; U)\right) \rightarrow$ $\mathbb{C}\left([0, T] ; \mathscr{L}^{2}(\Omega ; U)\right)$ defined by

$$
\begin{aligned}
\Phi(x)(t)= & S(t) x_{0}+\int_{0}^{t} S(t-s) \mathscr{F}(x)(s) d s \\
& +\int_{0}^{t} S(t-s) g(s) d \beta^{H}(s), \quad 0 \leq t \leq T .
\end{aligned}
$$

The first integral on the right side of (13) is taken in the Bochner sense, while the second is defined in Section 2. The operator $\Phi$ satisfies the following properties.

Lemma 9. Assume that (H1)-(H5) hold. Then, $\Phi$ is a welldefined, continuous map.

Proof. Using the discussion in Section 2 and the properties of $x$, one can see that for any $x \in \mathbb{C}\left([0, T] ; \mathscr{L}^{2}(\Omega ; U)\right)$, $\Phi(x)(t)$ is a well-defined stochastic process, for each $0 \leq$ $t \leq T$. In order to verify the continuity of $\Phi$ on $[0, T]$, let $z \in \mathbb{C}\left([0, T] ; \mathscr{L}^{2}(\Omega ; U)\right)$ and consider $0 \leq t^{*} \leq T$ and $|h|$ sufficiently small. Observe that

$$
\begin{gathered}
E\left\|\Phi(z)\left(t^{*}+h\right)-\Phi(z)\left(t^{*}\right)\right\|_{U}^{2} \\
\leq 3\left[E\left\|\left[S\left(t^{*}+h-s\right)-S\left(t^{*}-s\right)\right] x_{0}\right\|_{U}^{2}\right. \\
+E \| \int_{0}^{t^{*}+h} S\left(t^{*}+h-s\right) \mathscr{F}(z)(s) d s \\
\quad-\int_{0}^{t^{*}} S\left(t^{*}-s\right) \mathscr{F}(z)(s) d s \|_{U}^{2} \\
+E \| \int_{0}^{t^{*}+h} S\left(t^{*}+h-s\right) g(s) d \beta^{H}(s) \\
\left.\quad-\int_{0}^{t^{*}} S\left(t^{*}-s\right) g(s) d \beta^{H}(s) \|_{U}^{2}\right] \\
=3 \sum_{i=1}^{3}\left\|I_{i}\left(t^{*}+h\right)-I_{i}\left(t^{*}\right)\right\| .
\end{gathered}
$$

The semigroup property enables us to write

$$
\begin{aligned}
\left\|I_{1}\left(t^{*}+h\right)-I_{1}\left(t^{*}\right)\right\| & =E\left\|\left(\left(S\left(t^{*}+h\right)-S\left(t^{*}\right)\right) x_{0}\right)\right\|_{U}^{2} \\
& =E\left\|\left(S(h)\left(S\left(t^{*}\right) x_{0}\right)-S\left(t^{*}\right) x_{0}\right)\right\|_{U}^{2} .
\end{aligned}
$$

So, the strong continuity of $S(\cdot)$ implies that the right side of (15) goes to 0 as $|h| \rightarrow 0$. Next, using the Hölder inequality with $(\mathrm{H} 2)$ yields

$$
\begin{aligned}
& E\left\|\int_{t^{*}}^{t^{*}+h} S\left(t^{*}+h-s\right) \mathscr{F}(z)(s) d s\right\|_{U}^{2} \\
& \quad \leq 2 M_{\mathscr{F}}^{2} M_{S}^{2} h^{2}\left[1+\|z\|_{\mathbb{C}}^{2}+\|\mathscr{F}(0)\|_{\mathbb{C}}^{2}\right]
\end{aligned}
$$

which clearly goes to 0 as $|h| \rightarrow 0$. Also, the strong continuity of $S(\cdot)$ with (H2) enables us to conclude, with the help of the dominated convergence theorem, that

$$
E\left\|\int_{0}^{t^{*}}\left[S\left(t^{*}+h-s\right)-S\left(t^{*}-s\right)\right] \mathscr{F}(z)(s) d s\right\|_{U}^{2} \longrightarrow 0
$$

as $|h| \rightarrow 0$. Consequently, since $\left\|I_{2}\left(t^{*}+h\right)-I_{2}\left(t^{*}\right)\right\|$ is dominated by the expressions in (16) and (17), both of which go to 0 as $|h| \rightarrow 0$, it follows that $\left\|I_{2}\left(t^{*}+h\right)-I_{2}\left(t^{*}\right)\right\| \rightarrow 0$ as $|h| \rightarrow 0$.

It remains to show that $\left\|I_{3}\left(t^{*}+h\right)-I_{3}\left(t^{*}\right)\right\| \rightarrow 0$ as $|h| \rightarrow$ 0 . Observe that

$$
\begin{aligned}
& \left\|I_{3}\left(t^{*}+h\right)-I_{3}\left(t^{*}\right)\right\| \\
& =E \| \int_{0}^{t^{*}+h} S\left(t^{*}+h-s\right) g(s) d \beta^{H}(s) \\
& \quad-\int_{0}^{t^{*}} S\left(t^{*}-s\right) g(s) d \beta^{H}(s) \|_{U}^{2} \\
& =E \| \int_{t^{*}}^{t^{*}+h} S\left(t^{*}+h-s\right) g(s) d \beta^{H}(s) \\
& \quad+\int_{0}^{t^{*}}\left[S\left(t^{*}+h-s\right)-S\left(t^{*}-s\right)\right] g(s) d \beta^{H}(s) \|_{U}^{2} \\
& \leq 2 E\left\|\sum_{j=1}^{\infty} \int_{t^{*}}^{t^{*}+h} S\left(t^{*}+h-s\right) g(s) e_{j} d \beta_{j}^{H}(s)\right\|_{U}^{2} \\
& \quad+2 E\left\|\int_{0}^{t^{*}}\left[S\left(t^{*}+h-s\right)-S\left(t^{*}-s\right)\right] g(s) d \beta^{H}(s)\right\|_{U}^{2}
\end{aligned}
$$

and that

$$
\begin{aligned}
E\left\|\sum_{j=1}^{\infty} \int_{t^{*}}^{t^{*}+h} S\left(t^{*}+h-s\right) g(s) e_{j} d \beta_{j}^{H}(s)\right\|_{U}^{2} \\
=E\left\|\sum_{j=1}^{\infty} \int_{0}^{h} S(u) g\left(t^{*}+h-u\right) e_{j} d \beta_{j}^{H}\left(t^{*}+h-u\right)\right\|_{U}^{2} .
\end{aligned}
$$


Using the property $E\left(\beta_{j}^{H}(s)-\beta_{j}^{H}(t)\right)^{2}=|t-s|^{2 H} v_{j}$ with $s=$ $t^{*}+h$ and $t=t^{*}$ enables us to conclude that the right side of (19) goes to 0 as $|h| \rightarrow 0$. The second term on the right side of (18) goes to 0 as $|h| \rightarrow 0$ by Lemma 8(ii). Thus, $\left\|I_{3}\left(t^{*}+h\right)-I_{3}\left(t^{*}\right)\right\| \rightarrow 0$ as $|h| \rightarrow 0$ when $g$ is a simple function. Since the set of all such simple functions is dense in $\mathfrak{L}(V ; U)$, a standard density argument can be used to extend this conclusion to a general bounded, measurable function $g$. This establishes the continuity of $\Phi$.

Finally, we assert that $\Phi\left(\mathbb{C}\left([0, T] ; \mathscr{L}^{2}(\Omega ; U)\right)\right) \quad \subset$ $\mathbb{C}\left([0, T] ; \mathscr{L}^{2}(\Omega ; U)\right)$. Successive applications of Hölder's inequality yields

$$
\begin{aligned}
& {\left[E\left\|\int_{0}^{t} S(t-s) \mathscr{F}(z)(s) d s\right\|_{U}^{2}\right]^{1 / 2}} \\
& \quad \leq T^{1 / 2} M_{S}\left[\int_{0}^{T}\|\mathscr{F}(z)(s)\|_{\mathscr{L}^{2}(\Omega ; U)}^{2} d s\right]^{1 / 2} \\
& \quad \leq T^{1 / 2} M_{S}\|\mathscr{F}(z)\|_{\mathscr{L}^{2}} .
\end{aligned}
$$

Subsequently, an application of (H2), together with Minkowski's inequality, enables us to continue the string of inequalities in (20) to conclude that

$$
\begin{aligned}
& {\left[E\left\|\int_{0}^{t} S(t-s) \mathscr{F}(z)(s) d s\right\|_{U}^{2}\right]^{1 / 2}} \\
& \quad \leq T^{1 / 2} M_{S}\left[M_{\mathscr{F}}\|z\|_{\mathbb{C}}+\|\mathscr{F}(0)\|_{\mathscr{L}^{2}}\right] .
\end{aligned}
$$

Taking the supremum over $[0, T]$ in (21) then implies that $\int_{0}^{t} S(t-s) \mathscr{F}(z)(s) d s \in \mathbb{C}\left([0, T] ; \mathscr{L}^{2}(\Omega ; U)\right)$, for any $z \in$ $\mathbb{C}\left([0, T] ; \mathscr{L}^{2}(\Omega ; U)\right)$. The other estimates can be established as above, and when used in conjunction with Lemma 8, one can readily verify that $\sup _{0 \leq t \leq T} E\|\Phi(z)(t)\|_{U}^{2}<\infty$, for any $z \in \mathbb{C}([0, T] ; U)$. Thus, we conclude that $\Phi$ is well defined, and the proof of Lemma 9 is complete.

Our first existence result is as follows.

Theorem 10. Assume that (H1)-(H5) hold. Then, (1) has a unique mild solution on $[0, T]$.

Proof. We know that $\Phi$ is well defined and continuous from Lemma 9. Let $\delta=1 /\left(M_{S}^{2} M_{\mathscr{F}}^{2}+1\right)$. We prove that $\Phi$ has a unique fixed point in $\mathbb{C}\left([0, \delta] ; \mathscr{L}^{2}(\Omega ; U)\right)$. To this end, let $x, y \in \mathbb{C}\left([0, \delta] ; \mathscr{L}^{2}(\Omega ; U)\right)$. Observe that (13) implies that

$$
(\Phi x)(t)-(\Phi y)(t)=\int_{0}^{t} S(t-s)[\mathscr{F}(x)(s)-\mathscr{F}(y)(s)] d s .
$$

Squaring both sides and taking the expectation in (22) yields, with the help of Young's inequality,

$$
\begin{aligned}
& E\|(\Phi x)(t)-(\Phi y)(t)\|_{U}^{2} \\
& \quad \leq E\left\|\int_{0}^{t} S(t-s)[\mathscr{F}(x)(s)-\mathscr{F}(y)(s)] d s\right\|_{U}^{2} .
\end{aligned}
$$

Taking the supremum over $[0, \delta]$ in (23) and applying reasoning similar to that which led to (16) yield

$$
\begin{aligned}
\| \Phi x- & \Phi y \|_{\mathbb{C}\left([0, \delta] ; \mathscr{L}^{2}(\Omega ; U)\right)} \\
& \leq\left\|\int_{0}^{t} S(t-s)[\mathscr{F}(x)(s)-\mathscr{F}(y)(s)] d s\right\|_{\mathbb{C}\left([0, \delta] ; \mathscr{L}^{2}(\Omega ; U)\right)} \\
& \leq M_{S} M_{\mathscr{F}} \delta^{1 / 2}\|x-y\|_{\mathbb{C}\left([0, \delta] ; \mathscr{L}^{2}(\Omega ; U)\right)} \\
& <\|x-y\|_{\mathbb{C}\left([0, \delta] ; \mathscr{L}^{2}(\Omega ; U)\right)},
\end{aligned}
$$

where the last inequality in (24) follows from the choice of $\delta$. Hence, $\Phi$ is a strict contraction on $[0, \delta]$ and so has a unique fixed point which coincides with a mild solution of (1) on $[0, \delta]$. Performing this same argument on $[\delta, 2 \delta],[2 \delta, 3 \delta]$, and so on enables us to construct in finitely many steps a unique piecewise-defined function in $\mathbb{C}\left([0, T] ; \mathscr{L}^{2}(\Omega ; U)\right)$ which is a unique mild solution of (1) on the original interval $[0, T]$. This completes the proof.

Next, we consider the following initial-value problem:

$$
\begin{gathered}
d x(t) \\
=\left(A x(t)+\int_{0}^{t} B(t, s) f_{1}(s, x(s)) d s+f_{2}(t, x(t))\right) d t \\
+g(t) d \beta^{H}(t), \quad 0 \leq t \leq T, \\
x(0)=x_{0},
\end{gathered}
$$

where $\{B(t, s): 0 \leq t \leq s \leq T\} \subset \mathfrak{B} \mathfrak{Q}(U)$ and $f_{i}:[0, T] \times U \rightarrow$ $U(i=1,2)$ satisfy the following conditions:

(H6) $\{B(t, s): U \rightarrow U \mid 0 \leq t \leq s \leq T\}$ is a collection of bounded linear operators for which there exists a positive constant $M_{B}$ such that

$$
\|B(t, s)\|_{\mathfrak{B R}(U)} \leq M_{B}, \quad \forall 0 \leq t \leq s \leq T .
$$

(H7) $f_{i}:[0, T] \times U \rightarrow U(i=1,2)$ is such that there exists a positive constant $M_{f_{i}}$ for which

$$
\begin{array}{r}
\left\|f_{i}(t, x)-f_{i}(t, y)\right\|_{U} \leq M_{f_{i}}\|x-y\|_{U} \\
\forall t \in[0, T], x, y \in U .
\end{array}
$$

Corollary 11. If (H1), (H4), (H5), (H6a), and (H6) hold, then (22) has a unique mild solution on $[0, T]$.

Proof. Define $\mathscr{F} \quad: \quad \mathbb{C}\left([0, T] ; \mathscr{L}^{2}(\Omega ; U)\right) \quad \rightarrow \quad \mathscr{L}^{2}((0, T) ;$ $\left.\mathscr{L}^{2}(\Omega ; U)\right)$ by

$$
\mathscr{F}(x)(t)=\int_{0}^{t} B(t, s) f_{1}(s, x(s)) d s+f_{2}(t, x(t)),
$$

$$
0 \leq t \leq T
$$


Standard computations involving properties of expectation and Hölder's inequality imply, with the help of (H6) and (H7), that, for all $x, y \in \mathbb{C}\left([0, T] ; \mathscr{L}^{2}(\Omega ; U)\right)$,

$$
\begin{gathered}
\|\mathscr{F}(x)-\mathscr{F}(y)\|_{\mathscr{L}^{2}}^{2} \\
\leq 2 \int_{0}^{T}\left[T M_{B}^{2} \int_{0}^{s} E\left\|f_{1}(\tau, x(\tau))-f_{1}(\tau, y(\tau))\right\|_{U}^{2} d \tau\right. \\
\left.+E\left\|f_{2}(s, x(s))-f_{2}(s, y(s))\right\|_{U}^{2}\right] d s \\
\leq 2 T\left[T M_{B}^{2} M_{f_{1}}^{2}+M_{f_{2}}^{2}\right]\|x-y\|_{\mathbb{C}}
\end{gathered}
$$

Thus, if we let $M_{\mathscr{F}}=2 T\left[T M_{B}^{2} M_{f_{1}}^{2}+M_{f_{2}}^{2}\right]$ in (H2), we can conclude from Theorem 10 that (25) has a unique mild solution on $[0, T]$.

We now develop existence results for (1) in which the Lipschitz condition on $\mathscr{F}$ is replaced by the combination of continuity and a sublinear growth condition. This is done at the expense of a compactness restriction on the semigroup. Precisely, we use the following assumptions instead:

(H8) $A$ generates a compact $C_{0}$-semigroup $\{S(t): t \geq 0\}$ on $U$

(H9) $\mathscr{F}: \mathbb{C}\left([0, T] ; \mathscr{L}^{2}(\Omega ; U)\right) \rightarrow \mathscr{L}^{2}\left((0, T) ; \mathscr{L}^{2}(\Omega ; U)\right)$ is a continuous map such that there exists positive constants $c_{1}$ and $c_{2}$ such that

$$
\|\mathscr{F}(x)\|_{\mathscr{L}^{2}} \leq c_{1}\|x\|_{\mathbb{C}}+c_{2}
$$

for all $x \in \mathbb{C}\left([0, T] ; \mathscr{L}^{2}(\Omega ; U)\right)$.

We begin by establishing certain compactness properties of the mapping $\Phi_{1}: \mathscr{L}^{2}\left((0, T) ; \mathscr{L}^{2}(\Omega ; U)\right) \rightarrow$ $\mathbb{C}\left([0, T] ; \mathscr{L}^{2}(\Omega ; U)\right)$ defined by

$$
\Phi_{1}(v)(t)=\int_{0}^{t} S(t-s) v(s) d s, \quad 0 \leq t \leq T
$$

The well definedness of this mapping is essentially a stochastic analog of Lemma 3.1 in [31] (where $S(t)$ plays the role of the resolvent operator) and its proof follows similarly by making the natural modifications.

Lemma 12. Assume that $\{S(t): 0 \leq t \leq T\}$ is a compact semigroup on $U$. Then, $\Phi_{1}$ is a compact map from $\mathscr{L}^{2}\left((0, T) ; \mathscr{L}^{2}(\Omega ; U)\right)$ into $\mathbb{C}\left([0, T] ; \mathscr{L}^{2}(\Omega ; U)\right)$.

Theorem 13. Assume that (H3), (H4), (H5), (H8), and (H9) hold. Then, (1) has at least one mild solution on $[0, T]$.

Proof. We use Schaefer's theorem to prove that $\Phi$ (as defined in (13)) has a fixed point. The well definedness of $\Phi$ under (H3), (H4), (H5), (H8), and (H9) can be established using reasoning similar to that employed in the proof of Theorem 10. To verify the continuity of $\Phi$, let $\left\{\mu_{n}\right\}_{n=1}^{\infty}$ be a sequence in
$\mathbb{C}\left([0, T] ; \mathscr{L}^{2}(\Omega ; U)\right)$ such that $\mu_{n} \rightarrow \mu$ as $n \rightarrow \infty$. Standard computations yield

$$
\begin{aligned}
& \left\|\Phi\left(\mu_{n}\right)-\Phi(\mu)\right\|_{\mathbb{C}} \\
& \quad \leq M_{S}\left(E\left(\int_{0}^{T}\left\|\mathscr{F}\left(\mu_{n}\right)(s)-\mathscr{F}(\mu)(s)\right\|_{U} d s\right)^{2}\right)^{1 / 2} \\
& \quad \leq M_{S} T^{1 / 2}\left\|\mathscr{F}\left(\mu_{n}\right)-\mathscr{F}(\mu)\right\|_{\mathscr{L}^{2}} .
\end{aligned}
$$

The continuity of $\mathscr{F}$ ensures that the right side of (32) goes to 0 as $n \rightarrow \infty$, thereby verifying the continuity of $\Phi$.

Next, let $\delta=1 /\left(3 M_{S}^{2} c_{1}^{2}+1\right)$. We will show that the set $\xi(\Phi)$, as defined in Theorem 1 with $\mathbb{C}\left([0, \delta] ; \mathscr{L}^{2}(\Omega ; U)\right)$ in place of $X$, is bounded. Let $v \in \xi(\Phi)$ and observe that, arguing as in (20), applications of the Hölder and Young inequalities (with (H8)) yield

$$
\delta^{1 / 2}\left(E \int_{0}^{\delta}\|\mathscr{F}(\mu)(s)\|_{U}^{2} d s\right)^{1 / 2} \leq \delta^{1 / 2}\left(c_{1}\|\mu\|_{\mathbb{C}}+c_{2}\right) .
$$

Also, from Lemma 8 we can infer that

$$
E\left\|\int_{0}^{t} S(t-s) g(s) d \beta^{H}(s)\right\|_{U}^{2} \leq C_{t} \sqrt{\sum_{j=1}^{\infty} v_{j}}
$$

Thus, we conclude that, for all $\mu \in \xi(\Phi)$ and $0 \leq t \leq \delta$,

$$
\begin{aligned}
\lambda\|\mu\|_{\mathbb{C}} \leq \sqrt{3}[ & M_{S}\left\|x_{0}\right\|_{\mathscr{L}_{0}^{2}(\Omega ; U)} \\
& \left.+M_{S} \delta^{1 / 2}\left(c_{1}\|\mu\|_{\mathbb{C}}+c_{2}\right)+C_{t} \sqrt{\sum_{j=1}^{\infty} v_{j}}\right] .
\end{aligned}
$$

Taking into account that $\lambda \geq 1$ and the choice of $\delta$, we conclude from (33) that $\|\mu\|_{\mathbb{C}} \leq \eta$, where $\eta$ is a constant independent of $\mu$ and $\lambda$. So, $\xi(\Phi)$ is bounded.

In order to apply Schaefer's theorem, it remains to show that $\Phi$ is compact. To this end, let $r>0$ and define $K_{r}=\{\mu \in$ $\left.\mathbb{C}\left([0, \delta] ; \mathscr{L}^{2}(\Omega ; U)\right):\|\mu\|_{\mathbb{C}} \leq r\right\}$. Using the notation of (13) and (31), we have

$$
\begin{array}{r}
\Phi(\mu)=S(\cdot) x_{0}+\Phi_{1}(\mathscr{F}(\mu)(\cdot))+\int_{0}^{\cdot} S(\cdot-s) g(s) d \beta^{H}(s), \\
\mu \in \mathbb{C}\left([0, \delta] ; \mathscr{L}^{2}(\Omega ; U)\right) .
\end{array}
$$

We assert that $\Phi\left(K_{r}\right)$ is precompact in $\mathbb{C}\left([0, \delta] ; \mathscr{L}^{2}(\Omega ; U)\right)$. Indeed, the fact that $\left\{\mathscr{F}(\mu): \mu \in K_{r}\right\}$ is a bounded subset of $\mathscr{L}^{2}\left((0, \delta) ; \mathscr{L}^{2}(\Omega ; U)\right)$ (cf. (H9)), it follows from Lemma 12 that the set $\left\{\Phi_{1}(\mathscr{F}(\mu)): \mu \in K_{r}\right\}$ is precompact in $\mathbb{C}\left([0, \delta] ; \mathscr{L}^{2}(\Omega ; U)\right)$. Since the set

$$
\left\{S(\cdot) x_{0}+\int_{0}^{\cdot} S(\cdot-s) g(s) d \beta^{H}(s): \mu \in K_{r}\right\}
$$


is trivially precompact, we conclude that $\Phi\left(K_{r}\right)$ is precompact in $\mathbb{C}\left([0, \delta] ; \mathscr{L}^{2}(\Omega ; U)\right)$. So, Schaefer's theorem implies that $\Phi$ has a fixed point $x \in \mathbb{C}\left([0, \delta] ; \mathscr{L}^{2}(\Omega ; U)\right)$ which is a mild solution to $(1)$ on $[0, \delta]$. Performing this same argument on $[\delta, 2 \delta],[2 \delta, 3 \delta]$, and so on enables us to construct in finitely many steps a piecewise-defined function in $\mathbb{C}\left([0, T] ; \mathscr{L}^{2}(\Omega ; U)\right)$, that is, a mild solution of (1) on the original interval $[0, T]$. This completes the proof.

Next, we state a corollary regarding (25) under the following assumptions on $f_{i}$ :

(H10) $f_{i}:[0, T] \times U \rightarrow U(i=1,2)$ satisfies the following:

(i) $f_{i}(t, \cdot): U \rightarrow U$ is continuous, for almost all $t \in[0, T]$

(ii) $f_{i}(\cdot, x):[0, T] \rightarrow U$ is strongly $\mathscr{G}_{t}$-measurable, for all $x \in U$;

(iii) There exist positive constants $a_{i, 1}$ and $a_{i, 2}$ such that

$$
\left\|f_{i}(t, x)\right\|_{U} \leq a_{i, 1}\|x\|_{U}+a_{i, 2},
$$

for almost all $t \in[0, T]$ and for all $x \in U$.

Corollary 14. If (H3), (H4), (H5), (H8), and (H10) hold, then (25) has at least one mild solution on $[0, T]$.

Proof. An argument similar to the one used in [32, Chapter 26, pg. 561] shows that (H10) guarantees the mapping $\widetilde{\mathscr{F}}$ : $\mathbb{C}\left([0, T] ; \mathscr{L}^{2}(\Omega ; U)\right) \rightarrow \mathscr{L}^{2}\left((0, T) ; \mathscr{L}^{2}(\Omega ; U)\right)$ defined in (28) is well defined and continuous. Routine calculations show that $\widetilde{\mathscr{F}}$ satisfies (H9) with $c_{1}=2 T\left(a_{1,1} M_{B} T^{3 / 2}+a_{2,1}\right)$ and $c_{2}=2 T\left(a_{1,2} M_{B} T^{3 / 2}+a_{2,2}\right)$. Consequently, (25) has at least one mild solution by Theorem 13 .

We can formulate a stronger version of Corollary 14 by replacing assumption (H10) by the following:

(H11) $f_{i}:[0, T] \times U \rightarrow U(i=1,2)$ satisfies (H10) (i) and (ii), and

(i) for each $k \in \mathbb{N}$, there exists $g_{i, k} \in$ $\mathscr{L}^{1}((0, T) ;(0, \infty))$ such that for almost all $t \in$ $(0, T)$,

$$
\sup _{\|x\|_{U} \leq k} E\left\|f_{i}(t, x)\right\|_{U}^{2} \leq g_{i, k}(t) ;
$$

(ii) $\underline{\lim }_{k \rightarrow \infty} k^{-2} \int_{0}^{T} g_{i, k}(s) d s=\alpha_{i}<\infty$.

Proposition 15. Assume that (H3), (H4), (H5), (H8), and (H11) hold. Then, (25) has at least one mild solution on $[0, T]$.

Proof. We use Schauder's fixed-point theorem to argue that $\Phi$ (as defined in (13) with $\mathscr{F}$ given by (28)) has a fixed point. The continuity and compactness follow by making slight changes to the proof of Theorem 13. Choose $\delta$ such that

$$
4 M_{S}\left[\delta^{1 / 2} \alpha_{2}^{1 / 2}+M_{B} \delta \alpha_{1}^{1 / 2}\right]<1 .
$$

For $n \in \mathbb{N}$, define the set $B_{n}=\left\{x \in \mathbb{C}\left([0, \delta] ; \mathscr{L}^{2}(\Omega ; U)\right)\right.$ : $\left.\|x\|_{\mathbb{C}} \leq n\right\}$. It remains to show that there exists an $n \in \mathbb{N}$ such that $\Phi\left(B_{n}\right) \subset B_{n}$. Suppose, by way of contradiction, that, for each $k \in \mathbb{N}$, there exists $u_{k} \in B_{k}$ such that $\Phi\left(u_{k}\right) \notin B_{k}$. Then,

$$
1 \leq \underline{\lim }_{k \rightarrow \infty} k^{-1}\left\|\Phi\left(u_{k}\right)\right\|_{\mathbb{C}} .
$$

Observe that

$$
\begin{gathered}
\left\|\Phi\left(u_{k}\right)\right\|_{\mathbb{C}} \\
\leq 4 M_{S}\left[\left\|x_{0}\right\|_{\mathscr{L}_{0}^{2}(\Omega ; U)}\right. \\
+\delta^{1 / 2}\left(\delta^{1 / 2} M_{B}\left(\iint_{0}^{\delta} E\left\|f_{1}\left(\tau, u_{k}(\tau)\right)\right\|_{U}^{2} d \tau d s\right)^{1 / 2}\right. \\
\left.+\left(\int_{0}^{\delta} E\left\|f_{2}\left(s, u_{k}(s)\right)\right\|_{U}^{2} d s\right)^{1 / 2}\right) \\
\left.+E\left\|\int_{0}^{\delta} S(t-s) g(s) d \beta^{H}(s)\right\|_{U}^{2}\right] .
\end{gathered}
$$

Note that for each $k \in \mathbb{N}, u_{k} \in B_{k}$ and hence, $\left\|u_{k}(s)\right\|_{U} \leq k$, for all $0 \leq s \leq \delta$. So, by (H11), there exists $g_{i, k}(i=1,2)$, $j_{k} \in \mathscr{L}^{1}((0, \delta) ;(0, \infty))$ such that, for almost all $0 \leq s \leq \delta$,

$$
E\left\|f_{i}\left(s, u_{k}(s)\right)\right\|_{U}^{2} \leq g_{i, k}(s), \quad(i=1,2),
$$

Using (43) in (42) yields

$$
\begin{aligned}
\left\|\Phi\left(u_{k}\right)\right\|_{\mathbb{C}} & \\
\leq 2 M_{S}[ & \left\|x_{0}\right\|_{\mathscr{L}_{0}^{2}(\Omega ; U)}+\delta^{1 / 2}\left(\int_{0}^{\delta} g_{2, k}(s) d s\right)^{1 / 2} \\
& +M_{B} \delta^{3 / 2}\left(\int_{0}^{\delta} g_{1, k}(s) d s\right)^{1 / 2} \\
& \left.+E\left\|\int_{0}^{\delta} S(t-s) g(s) d \beta^{H}(s)\right\|_{U}^{2}\right]
\end{aligned}
$$


and subsequently,

$$
\begin{aligned}
& \varliminf_{k \rightarrow \infty} k^{-1}\left\|\Phi\left(u_{k}\right)\right\|_{\mathbb{C}} \\
& \leq 2 M_{S} \underset{k \rightarrow \infty}{\lim _{k \rightarrow \infty}}\left[k^{-1}\left\|x_{0}\right\|_{\mathscr{L}_{0}^{2}(\Omega ; U)}+\delta^{1 / 2}\left(k^{-2} \int_{0}^{\delta} g_{2, k}(s) d s\right)^{1 / 2}\right. \\
& +M_{B} \delta^{3 / 2}\left(k^{-2} \int_{0}^{\delta} g_{1, k}(s) d s\right)^{1 / 2} \\
& \left.+2 k^{-1} E\left\|\int_{0}^{\delta} S(t-s) g(s) d \beta^{H}(s)\right\|_{U}^{2}\right] \\
& \leq 2 M_{S} \delta^{1 / 2}\left(\alpha_{2}^{1 / 2}+M_{B} \delta \alpha_{1}^{1 / 2}\right)<1,
\end{aligned}
$$

contradicting (41). Consequently, there is an $n_{0} \in \mathbb{N}$ such that $\Phi\left(B_{n_{0}}\right) \subset B_{n_{0}}$. Thus, Schauder's fixed point theorem guarantees the existence of $x \in B_{n_{0}}$ such that $\Phi(x)=x$, which is a mild solution of $(25)$ on $[0, \delta]$. Performing this same argument on $[\delta, 2 \delta],[2 \delta, 3 \delta]$, and so on enables us to construct in finitely many steps a piecewise-defined function in $\mathbb{C}\left([0, T] ; \mathscr{L}^{2}(\Omega ; U)\right)$, that is, a mild solution of (1) on the original interval $[0, T]$. This completes the proof.

\section{Convergence and Approximation Results}

Throughout this section we assume that $A, \mathscr{F}$, and $g$ satisfy (H1)-(H5).

For each $n \in \mathbb{N}$, consider a linear operator $A_{n}: D\left(A_{n}\right)(=$ $D(A)) \rightarrow U$ and mappings $\mathscr{F}_{n}: \mathbb{C}\left([0, T] ; \mathscr{L}^{2}(\Omega ; U)\right) \rightarrow$ $\mathscr{L}^{2}\left((0, T) ; \mathscr{L}^{2}(\Omega ; U)\right)$, and $g_{n}:[0, T] \rightarrow \mathfrak{L}(V ; U)$ satisfying the following conditions:

(H12) $A_{n}$ generates a $C_{0}$-semigroup $\left\{S_{n}(t): t \geq 0\right\}$ such that $\left\|S_{n}(t)\right\|_{\mathfrak{B Q}(U)} \leq M_{S} e^{\alpha t}$, for some $\alpha>0$ (independent of $n$ ), for each $n \in \mathbb{N}$, and $\left\|A_{n} x-A x\right\|_{U} \rightarrow 0$ as $n \rightarrow$ $\infty$, for each $x \in D(A)$;

(H13)

(i) $\left\|\mathscr{F}_{n}(x)-\mathscr{F}_{n}(y)\right\|_{\mathscr{L}^{2}} \leq M_{\mathscr{F}}\|x-y\|_{\mathbb{C}}$, for all $x, y \in \mathbb{C}\left([0, T] ; \mathscr{L}^{2}(\Omega ; U)\right) ;$

(ii) $\left\|\mathscr{F}_{n}(x)-\mathscr{F}(x)\right\|_{\mathscr{L}^{2}} \rightarrow 0$ as $n \rightarrow \infty$, for all $x \in \mathbb{C}\left([0, T] ; \mathscr{L}^{2}(\Omega ; U)\right)$;

(H14) $g_{n}:[0, T] \rightarrow \mathfrak{Q}(V ; U)$ is a bounded, strongly measurable mapping and $\left\|g_{n}(t)-g(t)\right\|_{\mathfrak{L}(V ; U)} \rightarrow 0$ as $n \rightarrow \infty$, for all $0 \leq t \leq T$.

(Here, the constant $M_{\mathscr{F}}$ is the same one appearing in (H2) and so is independent of $n$.)

Assume that (H1)-(H5) hold. Then, by Theorem 10, (1) has a unique mild solution $x \in \mathbb{C}\left([0, T] ; \mathscr{L}^{2}(\Omega ; U)\right)$. By virtue of (H4), (H12)-(H14), Theorem 10 implies that, for each $n \in \mathbb{N}$, the initial-value problem

$$
\begin{array}{r}
d x_{n}(t)=\left(A_{n} x_{n}(t)+\mathscr{F}_{n}\left(x_{n}\right)(t)\right) d t+g_{n}(t) d \beta^{H}(t), \\
0 \leq t \leq T,
\end{array}
$$

$$
x_{n}(0)=x_{0}
$$

has a unique mild solution $x_{n} \in \mathbb{C}\left([0, T] ; \mathscr{L}^{2}(\Omega ; U)\right)$.

Consider the following initial-value problem:

$$
\begin{array}{r}
d y_{n}(t)=\left(A_{n} y_{n}(t)+\mathscr{F}_{n}(x)(t)\right) d t+g_{n}(t) d \beta^{H}(t), \\
0 \leq t \leq T, \\
y_{n}(0)=x_{0}
\end{array}
$$

Since $x_{0}$ is a fixed element of $\mathscr{L}_{0}^{2}(\Omega ; U)$, a standard argument guarantees the existence of a unique mild solution $y_{n}$ of (47). We need the following lemma.

Lemma 16. If (H12)-(H14) hold, then $\left\|y_{n}-x\right\|_{\mathbb{C}} \rightarrow 0$ as $n \rightarrow$ $\infty$.

Proof. Using (H12) in conjunction with Theorem 4.1 in [24, pg. 46], we infer that $S_{n}(t) z \rightarrow S(t) z$ as $n \rightarrow \infty$, for all $z \in U$, uniformly in $t \in[0, T]$. Observe that

$$
\begin{aligned}
& E\left\|y_{n}(t)-x(t)\right\|_{U}^{2} \\
& \leq 4 E\left\|\left(S_{n}(t)-S(t)\right) x_{0}\right\|_{U}^{2} \\
& \quad+4 E \int_{0}^{t}\left\|S_{n}(t-s)\left(\mathscr{F}_{n}(x)(s)-\mathscr{F}(x)(s)\right)\right\|_{U}^{2} d s \\
& +4 E \int_{0}^{t}\left\|\left(S_{n}(t-s)-S(t-s)\right) \mathscr{F}(x)(s)\right\|_{U}^{2} d s \\
& +4 E \| \int_{0}^{t}\left[S_{n}(t-s)\left(g_{n}(s)-g(s)\right)\right. \\
& \left.\quad+\left(S_{n}(t-s)-S(t-s)\right) g(s)\right] d \beta^{H}(s) \|_{U}^{2}
\end{aligned}
$$

A standard argument invoking (H12) and (H13), involving the Trotter-Kato Theorem [28], can be used to conclude that each of the first three terms on the right side of (48) goes to 0 as $n \rightarrow \infty$. As for the fourth term, observe that

$$
\begin{aligned}
E \| \int_{0}^{t}\left[S_{n}(t-s)\left(g_{n}(s)-g(s)\right)\right. \\
\left.+\left(S_{n}(t-s)-S(t-s)\right) g(s)\right] d \beta^{H}(s) \|_{U}^{2} \\
\leq E\left\|\int_{0}^{t} S_{n}(t-s)\left(g_{n}(s)-g(s)\right) d \beta^{H}(s)\right\|_{U}^{2} \\
\quad+E\left\|\int_{0}^{t}\left(S_{n}(t-s)-S(t-s)\right) g(s) d \beta^{H}(s)\right\|_{U}^{2} .
\end{aligned}
$$


The uniform boundedness of $\left\{S_{n}(t): 0 \leq t \leq T, n \in \mathbb{N}\right\}$ (cf. (H12)) with (H14) guarantees that the supremum (over $[0, T]$ ) of the first term on the right side of (49) goes to 0 as $n \rightarrow \infty$. An argument in the spirit of the one used to verify Lemma 8 (ii) can be used to show the supremum (over $[0, T]$ ) of the second term in (49) and also goes to 0 as $n \rightarrow \infty$, as needed. This completes the proof.

The following is the first of our two main convergence results.

Theorem 17. If (H1)-(H5) and (H12)-(H14) hold and $4 \overline{M_{S}} T M_{\mathscr{F}}<1$, where $\overline{M_{S}}=M_{S} e^{\alpha T}$, then $\left\|x_{n}-x\right\|_{\mathbb{C}} \rightarrow 0$ as $n \rightarrow \infty$.

Proof. Let $y_{n}$ be the mild solution of (47). Observe that

$$
\begin{aligned}
& \left\|x_{n}(t)-x(t)\right\|_{U}^{2} \\
& \leq 2\left[\left\|x_{n}(t)-y_{n}(t)\right\|_{U}^{2}+\left\|y_{n}(t)-x(t)\right\|_{U}^{2}\right] \\
& \leq 2\left\{\left(\int_{0}^{t}\left\|S_{n}(t-s)\left(\mathscr{F}_{n}\left(x_{n}\right)(s)-\mathscr{F}_{n}(x)(s)\right)\right\|_{U} d s\right)^{2}\right. \\
& \left.+\left\|y_{n}(t)-x(t)\right\|_{U}^{2}\right\} .
\end{aligned}
$$

Taking the expectation, followed by taking square roots in (50), yields the following estimate after some computation

$$
\begin{aligned}
& \left\|x_{n}(t)-x(t)\right\|_{\mathscr{L}^{2}(\Omega ; U)} \\
& \leq 2\left\{2 T ^ { 1 / 2 } \left(\int_{0}^{T} E \| S_{n}(t-s)\right.\right. \\
& \left.\quad \times\left(\mathscr{F}_{n}\left(x_{n}\right)(s)-\mathscr{F}_{n}(x)(s)\right) \|_{U^{2}}^{2} d s\right)^{1 / 2} \\
& \left.+\left\|y_{n}(t)-x(t)\right\|_{\mathscr{L}^{2}(\Omega ; U)}\right\} .
\end{aligned}
$$

Observe that (H12) yields, with the help of Hölder's inequality,

$$
\begin{aligned}
& \left(\int_{0}^{T} E\left\|S_{n}(t-s)\left(\mathscr{F}_{n}\left(x_{n}\right)(s)-\mathscr{F}_{n}(x)(s)\right)\right\|_{U}^{2} d s\right)^{1 / 2} \\
& \leq T^{1 / 2} \overline{M_{S}}\left\|\mathscr{F}_{n}\left(x_{n}\right)-\mathscr{F}_{n}(x)\right\|_{\mathscr{L}^{2}} \\
& \leq T^{1 / 2} \overline{M_{S}} M_{\mathscr{F}}\left\|x_{n}-x\right\|_{\mathbb{C}} .
\end{aligned}
$$

Using (51) and (52) in (50) yields, after taking supremum over $[0, T]$,

$$
\frac{1}{2}\left(1-4 \overline{M_{S}} T M_{\mathscr{F}}\right)\left\|x_{n}-x\right\|_{\mathbb{C}} \leq\left\|y_{n}-x\right\|_{\mathbb{C}} .
$$

In view of $(\mathrm{H} 12)-(\mathrm{H} 14)$ and the fact that $1-4 \overline{M_{S}} T M_{\mathscr{F}}>$ 0 , we can apply Lemma 16 to conclude from (53) that $\left\|x_{n}-x\right\|_{\mathbb{C}} \rightarrow 0$ as $n \rightarrow \infty$. This completes the proof.
Now, let $P_{x}$ and $P_{x_{n}}$ denote the probability measures on $\mathbb{C}\left([0, T] ; \mathscr{L}^{2}(\Omega ; U)\right)$ induced by the mild solutions $x$ and $x_{n}$ of (1) and (46), respectively. Using Theorem 17, we will prove that $P_{x_{n}} \stackrel{w}{\rightarrow} P_{x}$ as $n \rightarrow \infty$, for a special subclass of initialvalue problems. Precisely, we have the following.

Theorem 18. Assume that (H1), (H3), (H4), and (H5) hold, in addition to the following:

(H15) $x_{0} \in \mathscr{L}_{0}^{4}(\Omega ; U)$;

$(\mathrm{H} 16) \mathscr{F}: \mathbb{C}\left([0, T] ; \mathscr{L}^{2}(\Omega ; U)\right) \rightarrow \mathscr{L}^{p}\left((0, T) ; \mathscr{L}^{2}(\Omega ; U)\right)$ (where $p \geq 4$ ) is such that there exists a positive constant $M_{\mathscr{F}}$ for which

$$
\begin{array}{r}
\|\mathscr{F}(x)-\mathscr{F}(y)\|_{\mathscr{L}^{p}} \leq M_{\mathscr{F}}\|x-y\|_{\mathbb{C}}, \\
\forall x, y \in \mathbb{C}\left([0, T] ; \mathscr{L}^{2}(\Omega ; U)\right) ;
\end{array}
$$

$(\mathrm{H} 17) \mathscr{F}_{n}: \mathbb{C}\left([0, T] ; \mathscr{L}^{2}(\Omega ; U)\right) \rightarrow \mathscr{L}^{p}\left((0, T) ; \mathscr{L}^{2}(\Omega ; U)\right)$ (where $p \geq 4$ ) is such that

(i) $\left\|\mathscr{F}_{n}(x)-\mathscr{F}_{n}(y)\right\|_{\mathscr{L}^{p}} \leq M_{\mathscr{F}}\|x-y\|_{\mathbb{C}}$, for all $x, y \in \mathbb{C}\left([0, T] ; \mathscr{L}^{2}(\Omega ; U)\right)$,

(ii) $\left\|\mathscr{F}_{n}(x)-\mathscr{F}(x)\right\|_{\mathscr{L}^{p}} \rightarrow 0$ as $n \rightarrow \infty$, for all $x \in$ $\mathbb{C}\left([0, T] ; \mathscr{L}^{2}(\Omega ; U)\right)$, where $M_{\mathscr{F}}$ is the constant defined in (H15);

(H18) The operators $A_{n}: D(A) \subset U \rightarrow U$ are bounded and linear.

If $1-{\overline{M_{S}}}^{2} T^{2 / q}{\overline{M_{\mathscr{F}}}}^{2}>0($ where $1 / p+1 / q=1)$, then $P_{x_{n}} \stackrel{w}{\rightarrow} P_{x}$ as $n \rightarrow \infty$.

Proof. We begin by showing that $\left\{P_{x_{n}}\right\}_{n=1}^{\infty}$ is relatively compact in $\mathbb{C}\left([0, T] ; \mathscr{L}^{2}(\Omega ; U)\right)$ by appealing to the Arzelá-Ascoli theorem. To this end, we will first show that there exists $\eta>0$ such that

$$
\sup _{n \in \mathbb{N}} \sup _{0 \leq t \leq T}\left\|x_{n}(t)\right\|_{\mathscr{L}^{2}(\Omega ; U)}=\eta<\infty .
$$

Note that $x_{n}$ is given by

$$
\begin{aligned}
x_{n}(t)= & S_{n}(t) x_{0}+\int_{0}^{t} S_{n}(t-s) \mathscr{F}_{n}\left(x_{n}\right)(s) d s \\
& +\int_{0}^{t} S_{n}(t-s) g_{n}(s) d \beta^{H}(s), \quad 0 \leq t \leq T .
\end{aligned}
$$

Observe that

$$
\left\|S_{n}(t) x_{0}\right\|_{\mathscr{L}^{2}(\Omega ; U)}^{2} \leq{\overline{M_{S}}}^{2}\left\|x_{0}\right\|_{\mathscr{L}_{0}^{2}(\Omega ; U)}^{2} .
$$

Likewise, (H16) guarantees the existence of a positive constant $\overline{M_{\mathscr{F}}}$ such that $\left\|\mathscr{F}_{n}(0)\right\|_{\mathscr{L}^{p}} \leq \overline{M_{\mathscr{F}}}$, for all $n$, so that a standard argument now yields

$$
\begin{aligned}
E\left\|\int_{0}^{t} S_{n}(t-s) \mathscr{F}_{n}\left(x_{n}\right)(s) d s\right\|_{U}^{2} \\
\quad \leq T^{2 / q}{\overline{M_{S}}}^{2}\left[M_{\mathscr{F}}^{2}\left\|x_{n}\right\|_{\mathbb{C}}^{2}+{\overline{M_{\mathscr{F}}}}^{2}\right] .
\end{aligned}
$$


Also, $\left\{E\left\|\int_{0}^{t} S_{n}(t-s) g_{n}(s) d \beta^{H}(s)\right\|_{U}^{2}: n \in \mathbb{N}\right\}$ is uniformly bounded because of (H12) and (H14) and the uniform boundedness of $\left\{g_{n}: n \in \mathbb{N}\right\}$ in $\mathfrak{L}(V ; U)$. Combining the estimates (57) and (58) and rearranging terms enable us to conclude from (56) that (55) holds because $1-{\overline{M_{S}}}^{2} T^{2 / q}{\overline{M_{\mathscr{F}}}}^{2}>0$ and all constants in (56)-(58) are independent of $n$.

Next, we establish the equicontinuity by showing $E\left\|x_{n}(t)-x_{n}(s)\right\|_{U}^{4} \rightarrow 0$ as $(t-s) \rightarrow 0$, for all $0 \leq s \leq t \leq T$, uniformly for all $n \in \mathbb{N}$. We estimate each term of the expression for $x_{n}(t)-x_{n}(s)$ separately. The boundedness of $\left\{\left\|A_{n}\right\|_{\mathcal{B Q}(U)}: n \in \mathbb{N}\right\}$ (as guaranteed by (H18)) ensures that

$$
M_{S A}=\sup _{n \in \mathbb{N}}\left\|S_{n}(\cdot) A_{n}\right\|_{\mathfrak{B Q}(U)}<\infty .
$$

Employing Theorem 2.4(d) in [28] and taking into account (H12), (H14), and (59), we conclude that

$$
\begin{aligned}
E\left\|\left[S_{n}(t)-S_{n}(s)\right] x_{0}\right\|_{U}^{4} & \leq T^{4 / 3} \int_{S}^{t} E\left\|S_{n}(\tau) A_{n} x_{0}\right\|_{U}^{4} d \tau \\
& \leq T^{4 / 3} M_{S A}^{4}\left\|x_{0}\right\|_{\mathscr{L}_{0}^{4}(\Omega ; U)}^{4}(t-s)^{2} .
\end{aligned}
$$

Next, observe that

$$
\begin{gathered}
\int_{0}^{t} S_{n}(t-\tau) \mathscr{F}_{n}\left(x_{n}\right)(\tau) d \tau-\int_{0}^{S} S_{n}(s-\tau) \mathscr{F}_{n}\left(x_{n}\right)(\tau) d \tau \\
=\int_{0}^{S}\left[S_{n}(t-\tau)-S_{n}(s-\tau)\right] \mathscr{F}_{n}\left(x_{n}\right)(\tau) d \tau \\
+\int_{S}^{t} S_{n}(t-\tau) \mathscr{F}_{n}\left(x_{n}\right)(\tau) d \tau
\end{gathered}
$$

Using (59), (H12), and (H13) when estimating each of the two integrals on the right side of (61) separately yields

$$
\begin{gathered}
E\left\|\int_{0}^{S}\left[S_{n}(t-\tau)-S_{n}(s-\tau)\right] \mathscr{F}_{n}\left(x_{n}\right)(\tau) d \tau\right\|_{U}^{4} \\
\leq T^{8 / 3} \int_{0}^{S} \int_{s-\tau}^{t-\tau} E\left\|S_{n}(w) A_{n} \mathscr{F}_{n}\left(x_{n}\right)(w)\right\|_{U}^{4} d w d \tau \\
\leq M_{S A}^{4} T^{11 / 3}\left[M_{\mathscr{F}}^{4}\left\|x_{n}\right\|_{\mathbb{C}}^{4}+\overline{M_{\mathscr{F}}}\right](t-s)^{(p-4) / p} .
\end{gathered}
$$

Next,

$$
\begin{gathered}
E\left\|\int_{0}^{t} S_{n}(t-\tau) g_{n}(\tau) d \beta^{H}(\tau)-\int_{0}^{S} S_{n}(s-\tau) g_{n}(\tau) d \beta^{H}(\tau)\right\|_{U}^{4} \\
\leq E \| \sum_{j=1}^{\infty}\left[\int_{0}^{t} S_{n}(t-\tau) g_{n}(\tau) e_{j} d \beta_{j}^{H}(\tau)\right. \\
\left.\quad-\int_{0}^{S} S_{n}(s-\tau) g_{n}(\tau) e_{j} d \beta_{j}^{H}(\tau)\right] \|_{U}^{4} \\
\leq E\left\|\sum_{j=1}^{\infty} \int_{0}^{s}\left[S_{n}(t-\tau)-S_{n}(s-\tau)\right] g_{n}(\tau) e_{j} d \beta_{j}^{H}(\tau)\right\|_{U}^{4} \\
+E\left\|\sum_{j=1}^{\infty} \int_{s}^{t} S_{n}(t-\tau) g_{n}(\tau) e_{j} d \beta_{j}^{H}(\tau)\right\|_{U}^{4}
\end{gathered}
$$

Using the uniform boundedness of $\left\{g_{n}: n \in \mathbb{N}\right\}$ in $\mathfrak{Q}(V ; U)$, one can argue as in Lemma 8 to show that the right side of (63) goes to 0 as $(t-s) \rightarrow 0$. We conclude from the above estimates that $E\left\|x_{n}(t)-x_{n}(s)\right\|_{U}^{4} \rightarrow 0$ as $(t-s) \rightarrow 0$, uniformly for $0 \leq s \leq t \leq T$ and $n \in \mathbb{N}$, as desired. Thus, the family $\left\{P_{x_{n}}\right\}_{n=1}^{\infty}$ is relatively compact in $\mathbb{C}\left([0, T] ; \mathscr{L}^{2}(\Omega ; U)\right)$ and hence tight (by Prokorhov's theorem [9]).

To finish the proof, we remark that Theorem 17 implies that the finite-dimensional joint distributions of $P_{x_{n}}$ converge weakly to those of $P$ (cf. Proposition 3). Hence, Theorem 4 ensures that $P_{x_{n}} \stackrel{w}{\rightarrow} P_{x}$ as $n \rightarrow \infty$. This completes the proof.

\section{Extension to the Second-Order Case}

Consider the abstract second-order stochastic Cauchy problem

$$
\begin{gathered}
d x^{\prime}(t)=\left(\mathscr{B} x^{\prime}(t)+\mathscr{C} x(t)+F(x)(t)\right) d t+G(t) d \beta^{H}(t), \\
0 \leq t \leq T, \\
x(0)=x_{0}, \quad x^{\prime}(0)=x_{1}
\end{gathered}
$$

in a real separable Hilbert space $\widetilde{U}$. Here, $\mathscr{B}: \widetilde{U} \rightarrow \widetilde{U}$ is a bounded linear operator; $\mathscr{C}: D(\mathscr{C}) \subset \widetilde{U} \rightarrow \widetilde{U}$ is a linear (possibly unbounded) operator for which $(-\mathscr{C})^{1 / 2}$ exists; $F$ and $G$ are mappings that satisfy $(\mathrm{H} 2)$ and $(\mathrm{H} 3)$, respectively; $\left\{\beta^{H}(t): t \geq 0\right\}$ is a $\widetilde{U}$-valued $\mathrm{fBm}$ with Hurst parameter $H \in(1 / 2,1)$; and $x_{0}, x_{1} \in \mathscr{L}_{0}^{2}(\Omega ; \widetilde{U})$.

We will convert (64) to a first-order system that, in turn, can be represented abstractly in the form (1). To this end, let

$$
\begin{gathered}
z_{1}(t)=x(t), \quad z_{1}(0)=x_{0}, \\
z_{2}(t)=x^{\prime}(t), \quad z_{2}(0)=x_{1} .
\end{gathered}
$$


Then,

$$
\begin{gathered}
d z_{1}(t)=z_{2}(t) d t \\
d z_{2}(t)=\left(\mathscr{B} z_{2}(t)+\mathscr{C} z_{1}(t)+F\left(z_{1}\right)(t)\right) d t+G(t) d \beta^{H}(t) .
\end{gathered}
$$

As such,

$$
\begin{gathered}
d\left[\begin{array}{l}
z_{1}(t) \\
z_{2}(t)
\end{array}\right] \\
=\left(\left[\begin{array}{ll}
0 & I \\
\mathscr{C} & 0
\end{array}\right]\left[\begin{array}{l}
z_{1}(t) \\
z_{2}(t)
\end{array}\right]+\left[\begin{array}{ll}
0 & 0 \\
0 & \mathscr{B}
\end{array}\right]\left[\begin{array}{l}
z_{1}(t) \\
z_{2}(t)
\end{array}\right]+\left[\begin{array}{c}
0 \\
F\left(z_{1}\right)(t)
\end{array}\right]\right) d t \\
+\left[\begin{array}{c}
0 \\
G(t)
\end{array}\right] d \beta^{H}(t), \quad 0 \leq t \leq T \\
{\left[\begin{array}{l}
z_{1}(0) \\
z_{2}(0)
\end{array}\right]=\left[\begin{array}{l}
x_{0} \\
x_{1}
\end{array}\right] .}
\end{gathered}
$$

The space $U=D\left(\mathscr{C}^{1 / 2}\right) \times \widetilde{U}$ is a Banach space when equipped with the usual graph norm. Define $X:[0, T] \rightarrow U, A:$ $D(A) \subset U \rightarrow U, B: D(B) \subset U \rightarrow U, g:[0, T] \rightarrow \mathfrak{L}(V ; U)$, and $F: \mathbb{C}\left([0, T] ; \mathscr{L}^{2}(\Omega ; U)\right) \rightarrow \mathscr{L}^{2}\left((0, T) ; \mathscr{L}^{2}(\Omega ; U)\right)$ by

$$
\begin{gathered}
X(t)=\left[\begin{array}{l}
z_{1}(t) \\
z_{2}(t)
\end{array}\right], \\
A=\left[\begin{array}{ll}
0 & I \\
\mathscr{C} & 0
\end{array}\right], \quad D(A)=D(\mathscr{C}) \times D\left(\mathscr{C}^{1 / 2}\right) \subset U, \\
B=\left[\begin{array}{cc}
0 & 0 \\
0 & \mathscr{B}
\end{array}\right], \quad D(B)=D\left(\mathscr{C}^{1 / 2}\right) \times \widetilde{U} \subset U, \\
g(t)=\left[\begin{array}{c}
0 \\
G(t)
\end{array}\right], \quad F(X)(t)=\left[\begin{array}{c}
0 \\
F\left(z_{1}\right)
\end{array}\right](t) .
\end{gathered}
$$

Since

$$
X(0)=\left[\begin{array}{l}
z_{1}(0) \\
z_{2}(0)
\end{array}\right]=\left[\begin{array}{l}
x_{0} \\
x_{1}
\end{array}\right],
$$

we can use these identifications to rewrite (64) abstractly in the form

$$
\begin{gathered}
d X(t)=((A+B) X(t)+\mathscr{F}(X)(t)) d t+g(t) d \beta^{H}(t), \\
0 \leq t \leq T, \\
X(0)=X_{0} .
\end{gathered}
$$

We assume that the following conditions are satisfied.

$(\mathrm{H} 19) \mathscr{B}: D(\mathscr{B}) \subset \widetilde{U} \rightarrow \widetilde{U}$ is a bounded linear operator.

$(\mathrm{H} 20) \mathscr{C}: D(\mathscr{C}) \subset \widetilde{U} \rightarrow \widetilde{U}$ generates a strongly continuous cosine family on $\widetilde{U}$ and $(-\mathscr{C})^{1 / 2}$ exists.

Since $\mathscr{B} \in \mathfrak{B} \mathfrak{Q}(U)$, it follows that $A+B$ generates a strongly continuous semigroup on $U$. As such, we can view (70) (and so, (64)) as a special case of (1). Theorem 10 can be applied directly to (70) under the same hypotheses to conclude that (64) has a unique mild solution $X \in \mathbb{C}\left([0, T] ; \mathscr{L}^{2}(\Omega ; U)\right)$.

\section{Applications}

Let $\mathscr{D}$ be a bounded domain in $\mathbb{R}^{N}$ with smooth boundary $\partial \mathscr{D}$ and consider the initial-boundary value problem

$$
\begin{aligned}
& x_{t}(t, z) \\
& =\Delta_{z} x(t, z) \\
& +\int_{0}^{t} a(t, s) f_{1}\left(s, x(s, z), \int_{0}^{s} k(s, \tau, x(\tau, z)) d \tau\right) d s \\
& +f_{2}(t, z) d \beta^{H}(t), \quad \text { a.e. on }(0, T) \times \mathscr{D} \text {, } \\
& x(0, z)=x_{0}(z), \quad \text { a.e. on } \mathscr{D}, \\
& x(t, z)=0, \quad \text { a.e. on }(0, T) \times \partial \mathscr{D},
\end{aligned}
$$

We consider (71) under the following conditions on the data:

(H22) $f_{1}:[0, T] \times \mathbb{R} \times \mathbb{R} \rightarrow \mathbb{R}$ satisfies the Caratheódory conditions (i.e., measurable in $(t, x)$ and continuous in the third variable), and

(i) $f_{i}(\cdot, 0,0) \in L^{2}(0, T)$;

(ii) there exists a positive constant $M_{f_{1}}$ such that

$\left|f_{1}\left(t, x_{1}, y_{1}\right)-f_{1}\left(t, x_{2}, y_{2}\right)\right| \leq M_{f_{1}}\left[\left|x_{1}-x_{2}\right|+\left|y_{1}-y_{2}\right|\right]$,

for all $x_{1}, x_{2}, y_{1}, y_{2} \in \mathbb{R}$ and almost all $t \in(0, T)$;

$(\mathrm{H} 23) f_{2}:[0, T] \times \mathscr{D} \rightarrow \mathfrak{Q}\left(\mathscr{L}^{2}(\mathscr{D})\right.$; $\left.\mathscr{L}^{2}(\mathscr{D})\right)$ is a bounded, strongly measurable mapping;

(H24) $a \in L^{2}\left((0, T)^{2}\right)$;

$(\mathrm{H} 25) k: Z \times \mathbb{R} \rightarrow \mathbb{R}$, where $Z=\{(t, s): 0 \leq t \leq s \leq T\}$, satisfies the Caratheódory conditions and there exists a positive constant $M_{k}$ such that

$$
\left|k\left(t, s, x_{1}\right)-k\left(t, s, x_{2}\right)\right| \leq M_{k}\left|x_{1}-x_{2}\right|,
$$

for all $x_{1}, x_{2} \in \mathbb{R}$ and almost all $(t, s) \in Z$.

Let $U=V=\mathscr{L}^{2}(\mathscr{D})$ and set

$$
A=\Delta_{z}, \quad D(A)=H^{2}(\mathscr{D}) \cap H_{0}^{1}(\mathscr{D}) .
$$

It is well known that $A$ generates a $C_{0}$-semigroup on $D(A)$ (see [28], Chapter 7).

Next, define

$$
\begin{gathered}
\mathscr{F}: \mathscr{C}\left([0, T] ; \mathscr{L}^{2}(\Omega ; U)\right) \longrightarrow \mathscr{L}^{2}\left((0, T) ; \mathscr{L}^{2}(\Omega ; U)\right), \\
f_{2}:[0, T] \times \mathscr{D} \longrightarrow \mathfrak{L}\left(\mathscr{L}^{2}(\mathscr{D}) ; \mathscr{L}^{2}(\mathscr{D})\right),
\end{gathered}
$$

respectively, by

$$
\begin{gathered}
F(x)(t, \cdot) \\
=\int_{0}^{t} a(t, s) f_{1}\left(s, x(s, \cdot), \int_{0}^{s} k(s, \tau, x(\tau, \cdot)) d \tau\right) d s, \\
g(t)(\cdot)=f_{2}(t, \cdot) .
\end{gathered}
$$


One can use (H22)-(H25) to verify that $F$ satisfies (H2) with

$$
M_{\mathscr{F}}=2 M_{f_{1}} T|a|_{L^{2}\left((0, T)^{2}\right)}\left(1+M_{k} T^{3}\right)^{1 / 2}
$$

and that $g$ is strongly measurable. Thus, (71) can be rewritten in the form (1) in $U$, with $A, \mathscr{F}$, and $g$ defined above so that an application of Theorem 10 immediately yields the following result.

Theorem 19. If (H22)-(H25) hold (with $M_{\mathscr{F}}$ given by (77)), then (71) has a unique mild solution $x \in \mathbb{C}([0, T]$; $\left.\mathscr{L}^{2}\left(\Omega ; \mathscr{L}^{2}(\mathscr{D})\right)\right)$.

Example 20. We now consider a modified version of (71) which constitutes a model related to the one in [12]. Precisely, let $\mathscr{D}=\mathbb{R}$ and consider the initial-boundary value problem given by

$$
\begin{aligned}
& \partial x(t, z) \\
& =\left(-\left(I-\Delta_{z}\right)^{\gamma / 2}\left(-\Delta_{z}\right)^{\alpha / 2} x(t, z)\right. \\
& \left.\quad+\int_{0}^{t} a(t, s) f_{1}\left(s, x(s, z), \int_{0}^{s} k(s, \tau, x(\tau, z)) d \tau\right) d s\right) \partial t \\
& +f_{2}(t, z) d \beta^{H}(t), \quad \text { a.e. on }(0, T) \times \mathscr{D}, \\
& x(t, z)=0, \quad \text { a.e. on }(0, T) \times \partial \mathscr{D}, \\
& x(0, z)=x_{0}(z), \quad \text { a.e. on } \mathscr{D} .
\end{aligned}
$$

The operator $\left(-\Delta_{z}\right)^{\alpha / 2}$ is defined by

$$
\begin{gathered}
\left(-\Delta_{z}\right)^{\alpha / 2} h(z)=\frac{1}{\sqrt{2 \pi}} \int_{\mathbb{R}} e^{i z y}|y|^{\alpha} \widehat{h}(y) d y, \\
D\left(\left(-\Delta_{z}\right)^{\alpha / 2}\right) \\
=\left\{h \in \mathscr{L}_{w}^{2}(\mathbb{R}): h,|y|^{\alpha} \widehat{h}(y) \in \mathscr{L}^{1}(\mathbb{R}) \cap \mathscr{L}^{2}(\mathbb{R}),\right. \\
\left.\frac{1}{\sqrt{2 \pi}} \int_{\mathbb{R}} e^{i z y}|y|^{\alpha} \widehat{h}(y) d y \in \mathscr{L}_{w}^{2}(\mathbb{R})\right\},
\end{gathered}
$$

where $\widehat{h}$ denotes the Fourier transform of $h$, and the space $\mathscr{L}_{w}^{2}(\mathbb{R})$ is given by

$$
\begin{aligned}
\mathscr{L}_{w}^{2}(\mathbb{R})=\{h: h \text { is measurable, } \\
\left.\qquad h \|_{\mathscr{L}_{w}^{2}(\mathbb{R})}=\int_{\mathbb{R}}|h(z)|^{2} w(z) d z<\infty\right\},
\end{aligned}
$$

where $w(z)=\left(1+z^{2}\right)^{-\xi / 2}$, for $\xi>1$. Also, the operator $\left(I-\Delta_{z}\right)^{\gamma / 2}$ is defined by

$$
\begin{gathered}
\left(I-\Delta_{z}\right)^{\gamma / 2} h(z)=\frac{1}{\sqrt{2 \pi}} \int_{\mathbb{R}} e^{i z y}\left(1+y^{2}\right)^{\gamma / 2} \widehat{h}(y) d y, \\
D\left(\left(I-\Delta_{z}\right)^{\gamma / 2}\right) \\
=\left\{h \in \mathbb{Q}_{w}^{2}(\mathbb{R}): h,\left(1+y^{2}\right)^{\gamma / 2} \widehat{h}(y) \in \mathscr{L}^{1}(\mathbb{R}) \cap \mathscr{L}^{2}(\mathbb{R}),\right. \\
\left.\frac{1}{\sqrt{2 \pi}} \int_{\mathbb{R}} e^{i z y}\left(1+y^{2}\right)^{\gamma / 2} \widehat{h}(y) d y \in \mathscr{L}_{w}^{2}(\mathbb{R})\right\} .
\end{gathered}
$$

As shown in Proposition 1 of [12], the operator $-\left(I-\Delta_{z}\right)^{\gamma / 2}\left(-\Delta_{z}\right)^{\alpha / 2}$ generates a strongly continuous semigroup on $\mathscr{L}_{w}^{2}(\mathbb{R})$, assuming that $\alpha+\gamma>(\lambda-1) / 2$ and $\lambda<1$. As such, by taking $U=\mathscr{L}_{w}^{2}(\mathbb{R})$ and $V=\mathbb{R}$ and defining the operator $A=-\left(I-\Delta_{z}\right)^{\gamma / 2}\left(-\Delta_{z}\right)^{\alpha / 2}$, we can argue as in (71) to show that (78) has a unique mild solution $x \in \mathbb{C}\left([0, T] ; \mathscr{L}^{2}\left(\Omega ; \mathscr{L}_{w}^{2}(\mathbb{R})\right)\right)$.

Example 21. Let $\mathscr{D}$ be a bounded domain in $\mathbb{R}^{n}$ with smooth boundary $\partial \mathscr{D}$. Consider the following initial-boundary value problem:

$$
\begin{aligned}
& \partial\left(\frac{\partial x(t, \vec{z})}{\partial t}\right) \\
& =\left(\mathbf{B}\left(\frac{\partial x(t, \vec{z})}{\partial t}\right) \sum_{j=1}^{n} \sum_{k=1}^{n} \frac{\partial}{\partial z_{j}}\left(a_{j k}(\vec{z}) \frac{\partial x(t, \vec{z})}{\partial z_{j}}\right)\right) \partial t \\
& \quad+c(\vec{z}) x(t, \vec{z}) \partial t \\
& \quad+\left(f_{1}(t, x(t, \vec{z}))+\int_{0}^{t} b(t-s) f_{2}(s, x(s, \vec{z})) d s\right) \partial t \\
& \quad+f_{3}(t, \vec{z}) d \beta^{H}(t), \quad 0<t<T, \vec{z} \in \mathscr{D}, \\
& x(0, \vec{z})=x_{0}(\vec{z}), \quad \frac{\partial x(0, \vec{z})}{\partial t}=x_{1}(\vec{z}), \quad \text { a.e. on } \mathscr{D}, \\
& x(t, \vec{z})=0, \quad \text { a.e. on }(0, T) \times \partial \mathscr{D},
\end{aligned}
$$

where $\vec{z}=\left\langle z_{1}, \ldots, z_{n}\right\rangle \in \mathscr{D}, x_{0}(\cdot) \in D(A) \cap \mathscr{L}_{0}^{2}\left(\Omega ; \mathscr{L}^{2}(\mathscr{D})\right)$, and $x_{1}(\cdot) \in E \cap \mathscr{L}_{0}^{2}\left(\Omega ; \mathscr{L}^{2}(\mathscr{D})\right)$. Here, $a_{j k}: \mathscr{D} \rightarrow \mathbb{R}$ and $c: \mathscr{D} \rightarrow \mathbb{R}$ are bounded, strongly measurable mappings; and $f_{i}(i=1,2,3), \mathbf{B}$, and $b$ satisfy the following assumptions:

$(\mathrm{H} 26) f_{i}:[0, T] \times \mathbb{R} \rightarrow \mathbb{R}(i=1,2)$ satisfies the Carathéodory conditions, and

(i) $\left(f_{i}(\cdot, 0) \in L^{2}(0, T)\right.$;

(ii) there exists a positive constant $M_{f_{i}}$ such that

$$
\left|f_{i}(t, x)-f_{i}(t, y)\right| \leq M_{f_{i}}|x-y|,
$$

for all $x, y \in \mathbb{R}$, and almost all $t \in(0, T)$; 
(H27) $f_{3}:[0, T] \times \mathscr{D} \rightarrow \mathfrak{L}\left(\mathscr{L}^{2}(\mathscr{D}) ; \mathscr{L}^{2}(\mathscr{D})\right)$ is a bounded, strongly measurable mapping;

$(\mathrm{H} 28) \mathbf{B}: \mathscr{L}^{2}(\mathscr{D}) \rightarrow \mathscr{L}^{2}(\mathscr{D})$ is a bounded linear operator; (H29) $b \in \mathscr{L}^{2}\left((0, T)^{2}\right)$.

$$
\begin{aligned}
\text { Let } U & =V=\mathscr{L}^{2}(\mathscr{D}) \text { and define } A: U \rightarrow U \text { by } \\
A x(t, \cdot) & =\sum_{j=1}^{n} \sum_{k=1}^{n} \frac{\partial}{\partial z_{j}}\left(a_{j k}(\cdot) \frac{\partial x(t, \cdot)}{\partial z_{j}}\right)+c(\cdot) x(t, \cdot) .
\end{aligned}
$$

It is known that $A$ is a uniformly elliptic, denselydefined, symmetric, and self-adjoint operator which generates a strongly continuous cosine family on $U$ (see [27, p. 100]). Next, define $\mathscr{F} \quad: \quad \mathbf{C}\left([0, T] ; \mathscr{L}^{2}(\Omega ; U)\right) \rightarrow$ $\mathscr{L}^{2}\left((0, T) ; \mathscr{L}^{2}(\Omega ; U)\right)$ by

$$
\mathscr{F}(x)(t, \cdot)=f_{1}(t, x(t, \cdot))+\int_{0}^{t} b(t-s) f_{2}(s, x(s, \cdot)) d s .
$$

In view of (H26)-(H29), together with the Hölder and Young inequalities, one can verify that $\mathscr{F}$ satisfies (H2) with

$$
M_{\mathscr{F}}=2\left(M_{f_{1}} \sqrt{T}+M_{f_{2}}|b|_{\mathscr{L}^{2}\left((0, T)^{2}\right)}\right) .
$$

Hence, (82) can be written in the abstract form (64) in $U$ and so can be transformed into (1) via the procedure outlined in Section 5. As such, an application of Theorem 10 immediately yields the following result.

Theorem 22. If (H26)-(H29) are satisfied, then (82) has a unique mild solution $x \in \mathbb{C}\left([0, T] ; \mathscr{L}^{2}\left(\Omega ; \mathscr{L}^{2}(\mathscr{D})\right)\right)$.

\section{Conflict of Interests}

The authors declare that there is no conflict of interests regarding the publication of this paper.

\section{Acknowledgment}

The authors would like to express sincere gratitude to the anonymous referees who meticulously reviewed this paper and provided valuable feedback which greatly improved the presentation and strength of the results in this paper.

\section{References}

[1] P. J. Chen and M. E. Curtin, "On a theory of heat conduction involving two temperatures," Zeitschrift für Angewandte Mathematik und Physik, vol. 19, pp. 614-627, 1968.

[2] A. Fannjiang and T. Komorowski, "Fractional Brownian motions in a limit of turbulent transport," Annals of Applied Probability, vol. 10, no. 4, pp. 1100-1120, 2000.

[3] G. Gripenberg and I. Norros, "On the prediction of fractional Brownian motion," Journal of Applied Probability, vol. 33, no. 2, pp. 400-410, 1996.

[4] W. E. Leland, M. S. Taqqu, W. Willinger, and D. V. Wilson, "On the self-similar nature of Ethernet traffic (extended version)," IEEE/ACM Transactions on Networking, vol. 2, no. 1, pp. 1-15, 1994.
[5] S. O. Londen and J. A. Nohel, "Nonlinear Volterra integrodifferential equation occurring in heat flow," Journal of Integral Equations, vol. 6, pp. 11-50, 1984.

[6] K. Sobczyk, Stochastic Differential Equations With Applications to Physics and Engineering, Klüwer Academic Publishers, London, UK, 1991.

[7] B. B. Mandelbrot and J. W. Van Ness, "Fractional Brownian motions, fractional noises, and applications," SIAM Review, vol. 10, no. 4, pp. 422-437, 1968.

[8] N. U. Ahmed and C. D. Charalambous, "Filtering for linear systems driven by fractional Brownian motion," SIAM Journal on Control and Optimization, vol. 41, no. 1, pp. 313-330, 2006.

[9] P. Billingsley, Weak Convergence of Measures: Applications in Probability, SIAM, Bristol, UK, 1971.

[10] S. J. Lin, "Stochastic analysis of fractional Brownian motions," Stochastics and Stochastic Reports, vol. 55, pp. 121-140, 1995.

[11] D. Nualart and A. Rascanu, "Differential equations driven by fractional Brownian motion," Collectanea Mathematica, vol. 53, no. 1, pp. 55-81, 2002.

[12] V. V. Anh and W. Grecksch, "A fractional stochastic evolution equation driven by fractional Brownian motion," Monte Carlo Methods and Applications, vol. 9, no. 3, pp. 189-1199, 2003.

[13] T. E. Duncan, B. Pasik-Duncan, and B. Maslowski, "Fractional Brownian motion and stochastic equations in Hilbert space," Stochastics and Dynamics, vol. 2, no. 2, pp. 225-250, 2002.

[14] B. Maslowski and D. Nualart, "Evolution equations driven by a fractional Brownian motion," Journal of Functional Analysis, vol. 202, no. 1, pp. 277-305, 2003.

[15] D. A. Dawson and J. Gärtner, "Large deviations for the McKeanVlasov limit for weakly interacting diffusions," Stochastics, vol. 20, pp. 247-308, 1987.

[16] T. E. Govindan, "Autonomous semilinear stochastic Volterra integrodifferential equations in Hilbert spaces," Dynamic Systems and Applications, vol. 3, pp. 51-574, 1994.

[17] D. N. Keck and M. A. McKibben, "Abstract stochastic integrodifferential delay equations," Journal of Applied Mathematics and Stochastic Analysis, vol. 2005, no. 3, pp. 275-305, 2005.

[18] D. N. Keck and M. A. McKibben, "Functional integrodifferential stochastic evolution equations in Hilbert space," Journal of Applied Mathematics and Stochastic Analysis, vol. 16, no. 2, pp. 141-161, 2003.

[19] N. U. Ahmed, "Differential inclusions on Banach spaces with nonlocal state constraints," Nonlinear Functional Analysis and Its Applications, vol. 6, no. 3, pp. 395-3409, 2001.

[20] N. U. Ahmed and X. Ding, "A semilinear Mckean-Vlasov stochastic evolution equation in Hilbert space," Stochastic Processes and their Applications, vol. 60, no. 1, pp. 65-85, 1995.

[21] D. A. Dawson, "Critical dynamics and fluctuations for a meanfield model of cooperative behavior," Journal of Statistical Physics, vol. 31, no. 1, pp. 29-85, 1983.

[22] M. Nagasawa and H. Tanaka, "Diffusion with interactions and collisions between coloured particles and the propagation of chaos," Probability Theory and Related Fields, vol. 74, no. 2, pp. 161-198, 1987.

[23] H. Bergström, Weak Convergence of Measures, Academic Press, New York, NY, USA, 1982.

[24] N. Dunford and J. T. Schwarz, Linear Operators, Part I, Wiley Interscience, New York, NY, USA, 1958.

[25] W. Grecksch and C. Tudor, Stochastic Evolution Equations: A Hilbert Space Approach, Akademic, Berlin, Germany, 1995. 
[26] A. Ichikawa, "Stability of semilinear stochastic evolution equations," Journal of Mathematical Analysis and Applications, vol. 90, no. 1, pp. 12-44, 1982.

[27] H. Kunita, Stochastic Flows and Stochastic Differential Equations, Cambridge University Press, Cambridge, UK, 1990.

[28] A. Pazy, Semigroups of Linear Operators and Applications to Partial Differential Equations, Springer, New York, NY, USA, 1983.

[29] A. Granas and J. Dugundji, Fixed Point Theory, Springer, Berlin, Germany, 2003.

[30] W. Grecksch and V. V. Anh, "A parabolic stochastic differential equation with fractional Brownian motion input," Statistics and Probability Letters, vol. 41, no. 2, pp. 337-346, 1999.

[31] S. Aizicovici and K. B. Hannsgen, "Local existence for abstract semilinear Volterra integro-differential equations," Journal of Integral Equations and Applications, vol. 5, no. 3, pp. 299-2313, 1993.

[32] E. Zeidler, Nonlinear Functional Analysis and Its Applications II/B: Nonlinear Monotone Operators, Springer, New York, NY, USA, 1990. 


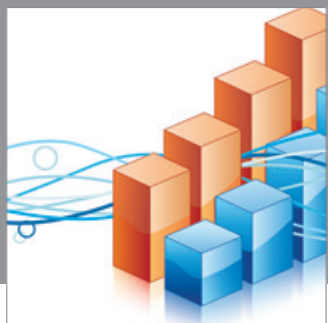

Advances in

Operations Research

mansans

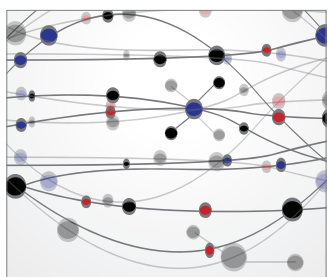

The Scientific World Journal
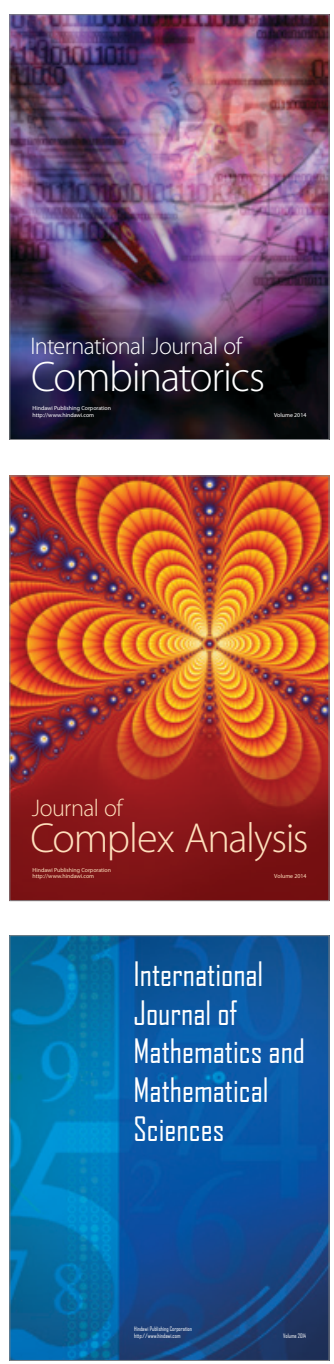
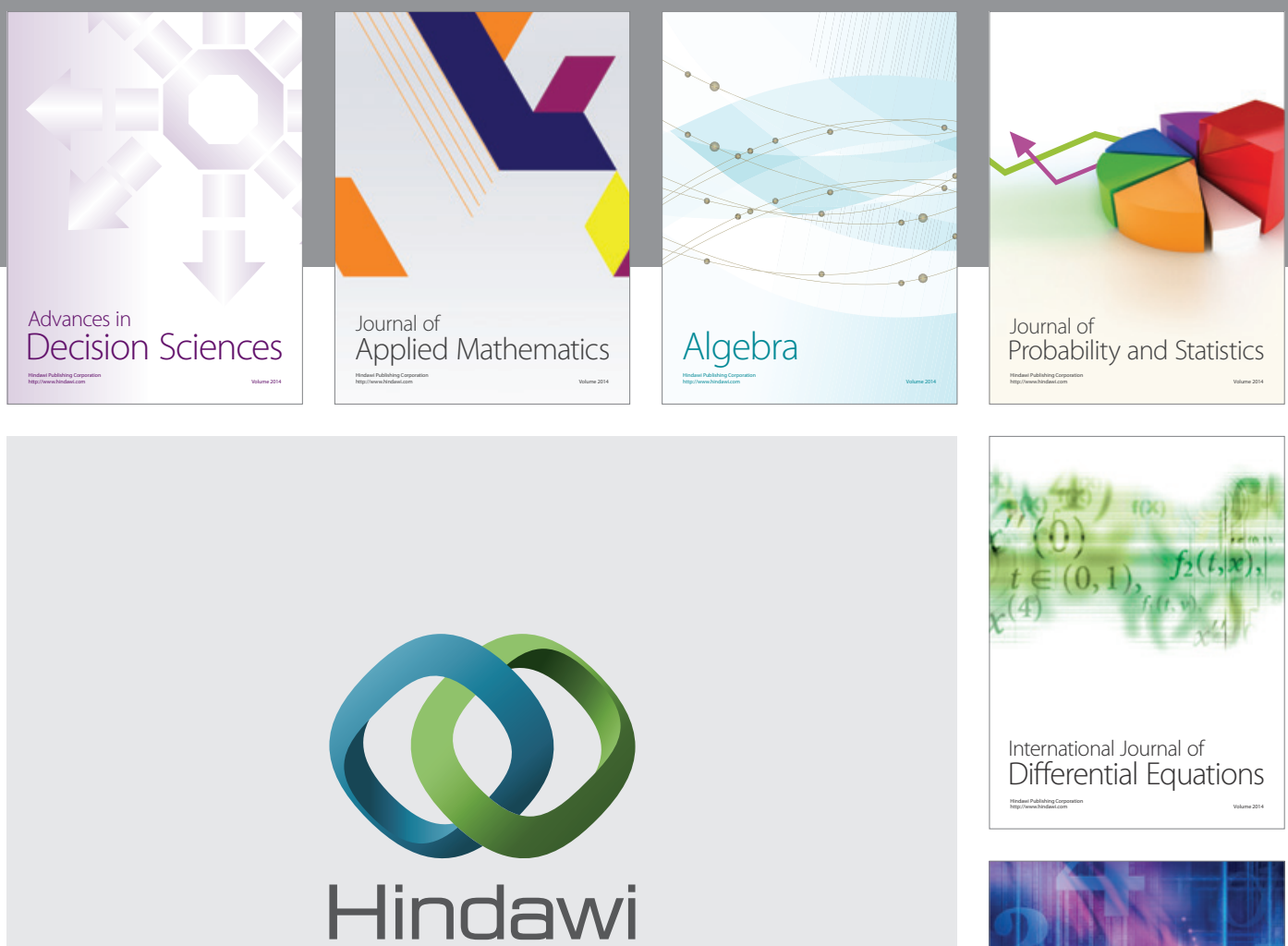

Submit your manuscripts at http://www.hindawi.com
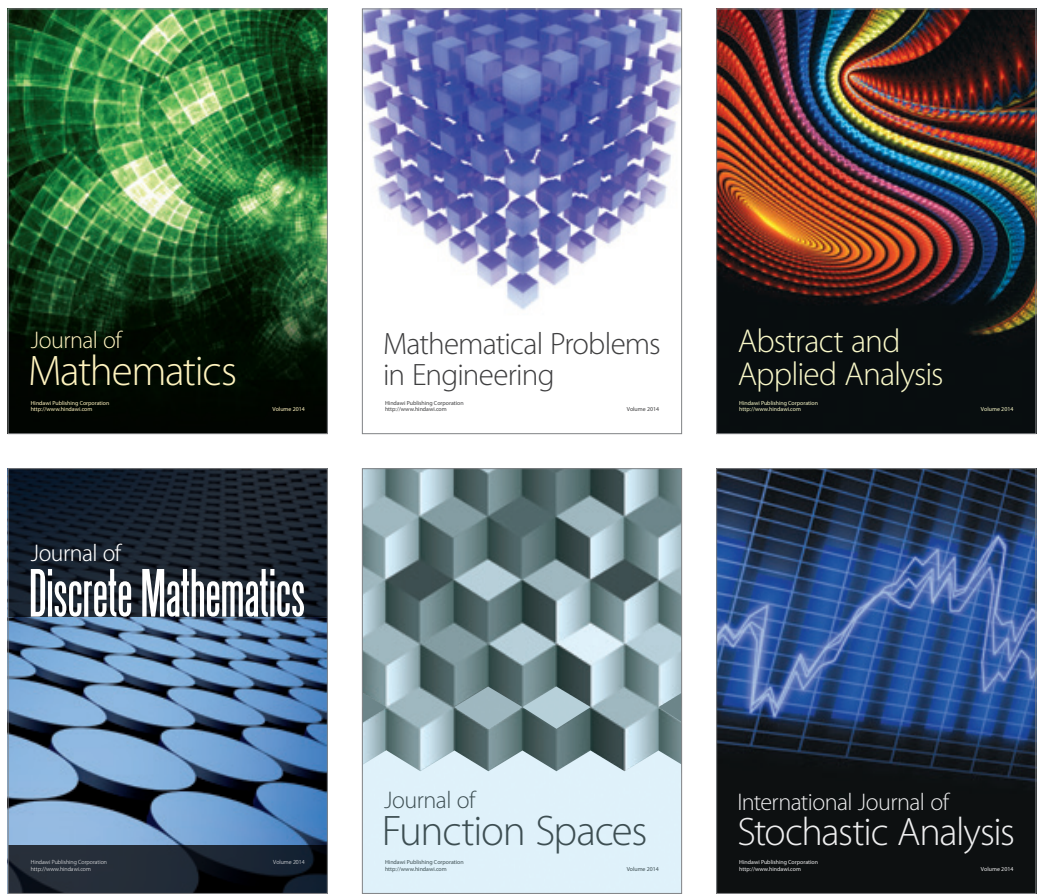

Journal of

Function Spaces

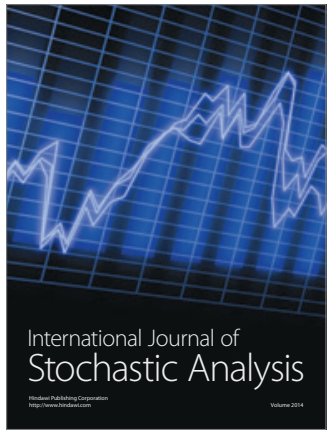

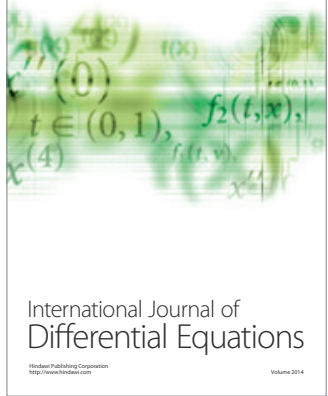
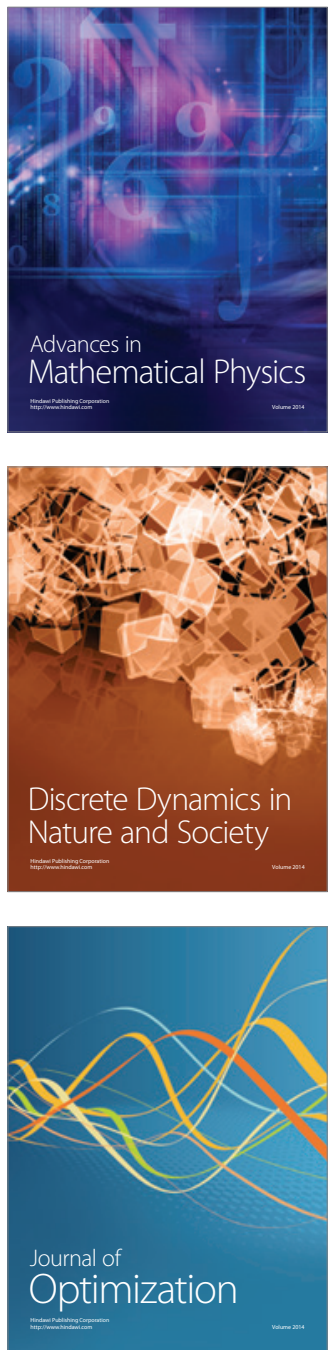